\title{
Die Entwicklung der Steuergerechtigkeitsideen und -prinzipien ab Mitte des 19. Jahrhunderts und ihre Realisierung in den Steuerreformen bis in das frühe 20. Jahrhundert
}

\subsection{Die Entwicklung der Steuergerechtigkeitsideen und -prinzipien ab Mitte des 19. Jahrhunderts}

Noch um die Jahrhundertmitte waren die klassischen Einwände gegen die allgemeine Einkommensteuer geläufig, die Ungerechtigkeit und auch die Unmöglichkeit einer allgemeinen Einkommensteuer wurden betont. ${ }^{540}$ Als der Vereinigte Preußische Landtag den Entwurf einer Einkommensteuer im Jahr 1847 beriet, die im Gegensatz zur britischen Income Tax die Selbsterklärung enthielt, wurde diese Neuerung als peinigend und demoralisierend verurteilt. Ebenfalls wurde erklärt, dass die Einkommensteuer die geheiligte Privatsphäre verletze und mit rücksichtsloser Zudringlichkeit die Familienverhältnisse entschleiern würde. ${ }^{541}$

540 Mann, Fritz Karl: Steuerpolitische Ideale, S. 242.

541 Ebd., S. 243. 


\subsubsection{Das Ideal der subjektiven allgemeinen Einkommensteuer}

Die Revolution von 1848/49 gab der Theorie der allgemeinen Einkommensteuer neue Kraft und Bedeutung. ${ }^{542}$ Die sich als fortschrittlich verstehenden Kräfte in Deutschland hielten es für ihre Pflicht, die Forderung nach einer allgemeinen Einkommensteuer auch theoretisch zu untermauern. Sie verteidigten „die verhasste Zwangsabgabe" als eine logische Folgerung aus dem klassischen Individualismus. ${ }^{543}$ Die sich verändernden politischen Verhältnisse und die verstärkt vorgetragenen Ideen bewirkten, dass man sich in der deutschen Literatur ein volles Jahrzehnt fast ausschließlich mit dem Problem der allgemeinen Einkommensteuer beschäftigte. ${ }^{544}$

Die Industrialisierung prägte die Epoche wie keine andere Entwicklung ein Jahrhundert geprägt hat. Die eher rückständigen deutschen Staaten entwickelten sich im europäischen Vergleich zu einer der führenden Industrienationen. $\mathrm{Zu}$ Beginn des 19. Jahrhunderts lag der Anteil der Gesamtbevölkerung, der in der Landwirtschaft beschäftigt war, bei knapp zwei Dritteln, um die Jahrhundertmitte fiel er dann auf etwa 55 Prozent und ein Vierteljahrhundert später auf knapp unter 50 Prozent. ${ }^{545} \mathrm{Im}$ Verlauf der Industrialisierung mit ihren neuen Produktionsverfahren, Produkten und Produktionsstätten schwand der Glaube, die steuerbaren Erträge der steuerbaren Objekte anhand von Kombinationen äußerer Merkmale hinreichend genau abschätzen zu können. ${ }^{546}$ Die sich ungerecht besteuert fühlenden Bürger beanstandeten die Belastung der fundierten Einkunftsarten ${ }^{547}$ aus den Quellen Grund und Boden, Gebäude und Gewerbebetriebe. Im württembergischen Parlament hieß es z. B. 1871/72, dass das geltende Ertragsteuersystem der württembergischen Verfassung von 1819 widerspräche, da die Gleichheit aller Staatsbürger nicht gewährleistet sei. ${ }^{548}$

542 Wilke, Gustav: Die Entwicklung der Theorie, S. 56.

543 Mann, Fritz Karl: Steuerpolitische Ideale, S. 244.

544 Meyer, Robert: Die Prinzipien der gerechten Besteuerung, S. $83 \mathrm{f}$.

545 Ziegler, Dieter: Das Zeitalter der Industrialisierung, S. 285.

546 Schremmer, Eckart: Über „gerechte Steuern“, S. 20.

547 Fundiertes Einkommen ist das auf Vermögen beruhende Einkommen. Unfundiertes Einkommen ist das nicht auf Vermögen beruhende, sondern aus Arbeit und Dienstleistungen stammende Einkommen; vgl. http://wirtschaftslexikon.gabler.de/Definition/ unfundiertes-einkommen.html (letzter Zugriff: 28.02.2017).

548 Schremmer, Eckart: Zusammenhänge zwischen Katastersteuersystem, Wirtschaftswachstum und Wirtschaftsstruktur im 19. Jahrhundert. Das Beispiel Württemberg: 1821-1877/1903, in: Bog, Ingomar u. a. (Hrsg.): Wirtschaftliche und soziale Strukturen 
Ein Weiteres kam hinzu: Ein Charakteristikum der durch Marktwirtschaft geprägten Gesellschaft des 19. Jahrhunderts war das Vordringen der Lohnarbeit und damit die Herausbildung der sozialen Klasse der Fabrikarbeiterschaft. Eine rasch wachsende Bevölkerungsgruppe wurde zu Lohn- und Gehaltsempfängern. Daneben wurde die alte, durch ständische Privilegien strukturierte Feudalgesellschaft von der wesentlich durch Besitz oder Nichtbesitz von Kapitalgütern strukturierten Marktgesellschaft abgelöst. ${ }^{549}$ Es gab immer mehr Personen, die ein Zinseinkommen aus Kapitalmarktpapieren erhielten. Beide Einkunftsarten wurden von den objektiven Ertragsteuern nicht und von den übrigen Steuern nur unzureichend - wenn überhaupt - erfasst. Die Nichterfassung dieser Einkünfte verstieß nicht nur gegen das Gerechtigkeitsprinzip, der Staat sah insbesondere ein neues Einnahmepotenzial, das er noch nicht richtig in Anspruch nehmen konnte. ${ }^{550}$

Der Durchbruch der Industrialisierung, zunächst beschränkt auf einige wenige Sektoren und Regionen bis hin zur Hochindustrialisierung, veränderte die gesamte Gesellschaft. Denn nicht nur eine neue Wirtschaftsordnung, die Marktwirtschaft, setzte sich im 19. Jahrhundert durch, sondern auch der Staat begann, sich von einem Rechts- zu einem Rechts-, Sozial-, Lenkungs- und Leistungsstaat zu wandeln. Die schwerfälligen und zunehmend als ungerecht empfundenen Katastersteuern waren für eine aktive Wirtschafts-, Sozial- und Finanzpolitik denkbar ungeeignet und darüber hinaus fiskalisch nicht ergiebig genug. ${ }^{551}$

Eine der bedeutendsten Persönlichkeiten der deutschen Freihandelsbewegung war der deutsche Nationalökonom, Publizist und Politiker John Prince-Smith. Prince-Smith forderte 1877, Eingriffe in die vom freien Wettbewerb hervorgebrachte Einkommensverteilung zu unterlassen, denn

eine allgemeine Einkommensteuer unterscheidet sich wesentlich von allen vorhin erwähnten Auflagen. Sie mischt sich gar nicht in die Produktion und Konsumtion, sondern hält sich lediglich an das Ergebniss. Sie lässt volle Freiheit des Erwerbens und nimmt erst vom Erworbenen. Und hierin liegt ihr grosser volkswirthschaftlicher Vorzug. Sie mindert, wie jede Besteuerung, die Fähigkeit, das Kapital zu mehren; aber sie richtet nicht, neben dem Schaden des Fortnehmens, noch durch die Art des Nehmens weiteren Schaden an, wie andere Steuern es thun. Sie erzeugt keinen Beweggrund, Produktion und Konsumtion anders einzurichten, als nach den rein

im saekularen Wandel. Festschrift für Wilhelm Abel zum 70. Geburtstag, Bd. 3, Hannover 1974, S. 679-706, S. 688 f.

549 Ziegler, Dieter: Das Zeitalter der Industrialisierung, S. 286.

550 Schremmer, Eckart: Über „gerechte Steuern“, S. 20.

551 Ebd., S. 20; Ziegler, Dieter: Das Zeitalter der Industrialisierung, S. 197-286. 
volkswirthschaftlichen Bedingungen für den höchsten Ertrag. Sie erzeugt also kein Bestreben, sie abzuwälzen oder ihr auszuweichen, denn beides ist untuhnlich. ${ }^{552}$

Schließlich wurde die liberale Ideologie zum Wegbereiter der Einkommensteuer, da die parlamentarische Demokratie ihr Steuerbewilligungsrecht am wirksamsten bei den direkten Steuern ausüben konnte. Die Volksvertretung konnte zwischen den Opfern der Steuerpflichtigen und den öffentlichen Leistungen des Staates vergleichen und damit ihren Willen der jeweiligen Regierung auferlegen. Die liberale Ideologie nahm die naturrechtliche Überlieferung vom Vorrang der direkten Steuern wieder auf und gab dem Plan einer allgemeinen Einkommensteuer auch ein politisches Fundament. ${ }^{553}$

Das in der Vergangenheit herrschende Äquivalenzprinzip der Besteuerung wurde - zumindest in der Theorie - allmählich vom Leistungsfähigkeitsprinzip verdrängt. Die Steuer sollte nicht mehr als eine Art Marktpreis festgesetzt, sondern nach der persönlichen Leistungsfähigkeit der Steuerträger bemessen werden. Diesem Ziel konnte die Einkommensteuer viel besser Rechnung tragen, als es die überkommenen Objektsteuern konnten. ${ }^{54}$ Die ganz auf das Individuum zugeschnittene steuerliche Leistungsfähigkeit und die darauf ausgerichtete Steuerlastverteilung galten den Autoren als das Höchstmaß an erreichbarer Gerechtigkeit. Aber auch wegen ihrer fiskalischen Ergiebigkeit, ihrer Anpassung an Struktur- und Wachstumsänderungen der Wirtschaft und ihrer geringen Erhebungskosten - nach Wegfall von Katastern und Einschätzungen - wurde die Einkommensteuer das neue Ideal der Besteuerung, sie wurde zur „Königin der Steuern“. 555

Das Ideal der subjektiven allgemeinen Einkommensteuer fasst Schremmer wie folgt zusammen: „Die entscheidenden Veränderungen der subjektiven Einkommensteuer gegenüber der objektiven Ertragsteuer liegen in der Verknüpfung von Steuergerechtigkeit, Gleichheit der Besteuerung und steuerlicher Leistungsfähigkeit." ${ }^{\text {556 }}$

552 Prince-Smith, John: Über die Abwälzung, in: Michaelis, Otto (Hrsg.): John Prince-Smiths Gesammelte Schriften. Zur Physiologie des Verkehrs, Staat und Volkshaushalt. Ueber das Denken. Zur Münzreform. Zur Lohnfrage. Die Sozialdemokratie, Bd. 1, Berlin 1877, S. 43-64, S. 60.

553 Mann, Fritz Karl: Steuerpolitische Ideale, S. 247 f.

554 Homburg, Stefan.: Allgemeine Steuerlehre, S. 42.

555 Schremmer, Eckart: Über „gerechte Steuern“, S. 21.

556 Ebd., S. 21. 


\subsubsection{Die individualistische Lehrmeinung}

Der im Revolutionsjahr 1848 von Johann Friedrich Mentzendorff gemachte Vorschlag einer „indirekten Reineinkommensteuer“, die ausschließlich auf das Reineinkommen der Grundbesitzer und gewerblichen Unternehmer bezogen war, denen jedoch die Überwälzung auf die übrigen Teile der Bevölkerung überlassen bleiben sollte, ${ }^{557}$ ist ohne Bedeutung für die weitere geschichtliche Entwicklung geblieben. Diese Lehre war in der Mitte des 19. Jahrhunderts heftigen Angriffen ausgesetzt.

Eduard Baumstark warf der allgemeinen Einkommensteuer in seiner im Jahr 1850 veröffentlichten Schrift „Zur Einkommensteuerfrage" ${ }^{\text {“558 }}$ vor, sie genüge weder dem Gesetze der Allgemeinheit noch dem Gesetze der Gleichheit. Er hielt die bestehenden Steuersysteme für prinzipiell gerechtfertigt; denn eine allgemeine Einkommensteuer sei nur unter ganz speziellen Bedingungen zu empfehlen: als Ersatz für noch schlechtere Steuern, als Notbehelf oder vorübergehend als Aushilfsteuer, und das auch nur dann, wenn der Zweck eine höhere Belastung der Wohlhabenden sei..$^{59}$

Lebhaft wandte sich auch Heinrich Ludwig Biersack in seinem 1850 erschienenen Werk „Ueber Besteuerung, ihre Grundsätze und ihre Ausführung “560 gegen diese Lehre ${ }^{561}$ und empfand die progressiven Steuern als verwerflich, „wenn sie einen socialistischen oder communistischen Zweck haben; wenn sie namentlich dazu dienen sollen, allmälig die in Ansehung des Besitzes von sachlichen Gütern bestehenden Verhältnisse aufzuheben. ${ }^{\text {"562 }}$

Trotz dieser negativen Stellungnahmen entwickelte sich eine Bewegung mit immer neuen Verteidigern, die die Theorie einer allgemeinen Einkommensteuer weiter zu vertiefen suchten. Schon im Revolutionsjahr 1848 ragte aus dem Schrifttum dieser Zeit das Buch „Die allgemeine Einkommensteuer als einzige gerechte direkte Abgabe ${ }^{“ 563}$ hervor, mit dem der preußische Landrat Karl von Sparre die Behauptung

557 Mentzendorff, Johann Friedrich: Neues Staatswirthschaftssystem zur Beförderung eines allgemeinen und dauernden Volkswohlstandes, IV. Theil, 1. Abtheilung, Magdeburg 1848, S. 36, $\$ 11$.

558 Baumstark, Eduard: Zur Einkommensteuerfrage, Greifswald 1850.

559 Meyer, Robert: Die Prinzipien der gerechten Besteuerung, S. 86.

560 Biersack, Heinrich Ludwig: Ueber Besteuerung, ihre Grundsätze und ihre Ausführung, Frankfurt am Main 1850.

561 Wilke, Gustav: Die Entwicklung der Theorie, S.57.

562 Zitiert nach: Meyer, Robert: Die Prinzipien der gerechten Besteuerung, S. 89 f.

563 Sparre, Karl von: Die allgemeine Einkommensteuer als einzige gerechte direkte Abgabe, Gießen 1848. 
aufstellte, dass dieser Sachverhalt aus Theorie und Erfahrung nachgewiesen wäre. ${ }^{564}$ Ebenso wie bereits Murhard im Jahr 1834 berücksichtigte er bei der Ermittlung des Einkommens die persönlichen Verhältnisse des Steuersubjekts. Im Gegensatz zu Murhard wollte er jedoch nicht den absolut notwendigen Unterhalt, sondern den standesgemäßen Lebensbedarf des Steuerpflichtigen und seiner Familie von seinem Gesamteinkommen abziehen. ${ }^{565}$ Er prophezeite der Einkommensteuer, dass sie nach und nach alle direkten Abgaben in sich aufnehmen und in Verbindung mit den indirekten Steuern die alleinige Grundlage der Staatsbedarfsbefriedigung sein würde. ${ }^{566}$

In der Literatur blieb es zunächst bei der Anerkennung der Besteuerung nach dem Einkommen, die jedoch auf verschiedene Weise begründet wurde. Robert von Mohl erschien die einzige direkte Einkommensteuer als die Verwirklichung einer gerechten Besteuerung, weil die Grundlage der Besteuerung nur die im Einkommen zum Ausdruck gelangende Leistungsfähigkeit des einzelnen Staatsbürgers sein könne. ${ }^{567}$ Während von Mohl den Grund der Steuer in der Notwendigkeit des Staatsbedarfs erblickte und in diesem Sinn auch die Beschränkung der Steuerpflicht sah, bekannten sich andere Autoren entweder offen zu dem Prinzip der Leistung und Gegenleistung oder standen dieser Auffassung sehr nahe. ${ }^{568}$ In der im Jahr 1855 in Zürich erschienenen Dissertation „Ueber die Einkommensteuer “569 befürwortete Carl Wilhelm von Graffenried eine allgemeine, auf Selbstschätzung beruhende Einkommensteuer. Auch er forderte eine völlige Befreiung für den zum Lebensunterhalt notwendigen Teil des Einkommens von jeder Besteuerung. Von Graffenried wandte sich gegen die Heranziehung der Konsumbesteuerung als Ergänzung der Erträgnisse einer einzigen Einkommensteuer, wie sie Murhard und Sparre forderten, da diese ein mehr schädliches als nützliches Vermächtnis des Merkantilismus darstelle. ${ }^{570}$

Max Wirth, der die Steuer als Entgelt für Staatsleistungen definierte, vertrat den Standpunkt, dass die Verteilung der Steuern nach dem Grundsatz von Leistung und Gegenleistung zu einer progressiven Steuer und damit bis zur Absurdität führe,

564 Wilke, Gustav: Die Entwicklung der Theorie, S. 56; Mann, Fritz Karl: Steuerpolitische Ideale, S. 252 mit Anm. 3.

565 Wilke, Gustav: Die Entwicklung der Theorie, S. 56.

566 Meyer, Robert: Die Prinzipien der gerechten Besteuerung, S. $88 \mathrm{f}$.

567 Mohl, Robert von: Encyklopädie der Staatswissenschaften, Tübingen 1859, S. 368.

568 Meyer, Robert: Die Prinzipien der gerechten Besteuerung, S. $100 \mathrm{f}$.

569 Graffenried, Carl Wilhelm von: Ueber die Einkommensteuer: ein staatswissenschaftlicher Versuch, Zürich 1855.

570 Wilke, Gustav: Die Entwicklung der Theorie, S. 58. 
da sie das ganze Einkommen verschlinge. Eine mäßige Progression hielt er jedoch für angemessen. Er lehnte die Steuerfreiheit des Existenzminimums ab, da es nur billig und gerecht sei, wenn auch der Ärmste einen - wenn auch nur geringen Anteil - an den Staatslasten trage, da auch er den Schutz des Staates genieße. Den direkten Steuern gab Wirth vor den indirekten Steuern den Vorzug und hoffte, dass die Einkommensteuer einen immer größeren Teil der Steuern in sich aufnähme. $\mathrm{Da}$ Wirth die direkte persönliche Einkommensteuer für eine gerechte Abgabe hielt, empfahl er aus psychologischen Gründen, diese zunächst neben den anderen Steuern einzuführen, um dann mithilfe der Einkommensteuer die übrigen Steuern ganz zu verdrängen. ${ }^{571}$

Georg Friedrich Wilhelm Roscher lehnte die Einkommensteuer als einzige Steuer ab, wollte jedoch diese als gleichberechtigte Steuer in das System der Steuern einfügen. Im Hinblick auf die verhältnismäßig hohe Belastung der unteren Klassen mit der Besteuerung der Konsumtion empfahl er, die Einkommensteuer auf das Einkommen der wohlhabenden Klassen mit einem niedrigeren Satz umzulegen. In Kriegs- oder anderen Notfällen mochte seiner Ansicht nach die Einkommensteuer bis zur vollen Deckung des Bedarfs anschwellen. ${ }^{572}$

Auch Lorenz von Stein wird zu den Anhängern der individualistischen Auffassung gezählt, obwohl er sich gegen die Anerkennung des Grundsatzes der Leistung und Gegenleistung verwehrte. Berücksichtigt werden muss dabei jedoch, dass sich seine Auffassung in seinem „Lehrbuch der Finanzwissenschaften ${ }^{\text {“573 }}$ - in nahezu zwei Jahrzehnte auseinanderliegenden Auflagen - stark veränderte. ${ }^{574}$ Von Stein betrachtete das Steuerwesen als einen Prozess im Leben der menschlichen Gemeinschaft und leitete daraus, dass der Staat die uneingeschränkte Bedingung für das persönliche Leben des Einzelnen sei, ein absolutes wirtschaftliches Recht des Staates ab und führte dazu aus, dass ,sich der souveräne Wille des Staats auch für sein finanzielles Leben aus der organisirten Gemeinschaft aller Willen bilden" müsse.

Aus dem Verständnis dieser Wechselwirkung zwischen der Souveränität des verfassungsgemäßen organisirten Staats und der freien Entwicklung seiner Angehörigen

571 Wirth, Max: Grundzüge der Nationalökonomie, 2. Aufl., Bd. 2, Köln 1861, S. 474 f.

572 Roscher, Wilhelm: System der Finanzwissenschaft. Ein Hand- und Lesebuch für Geschäftsmänner und Studierende (1854), Bd. 4, Erste Abtheilung, 2. Aufl., Stuttgart $1886, \S 74$.

573 Stein, Lorenz von: Lehrbuch der Finanzwissenschaft, Erster Theil: Die Finanzverfassung Europas. Mit specieller Vergleichung Englands, Frankreichs, Deutschlands, Oesterreichs, Italiens, Rußlands und anderer Länder, 5. Aufl., Leipzig 1885, ND Hildesheim/ New York 1975, S. 53 f.

574 Meyer, Robert: Die Prinzipien der gerechten Besteuerung, S. 104. 
entsteht dann für das wirthschaftliche Leben aus dem Finanzwesen die Finanzwirtschaft des Staats [...], welche für die Einheit der souveränen Persönlichkeit des Staats und der freien Entwickelung seiner Angehörigen

sorgen würde. ${ }^{575}$ Damit hatte von Stein die Frage nach der Gerechtigkeit aus seiner Darstellung eliminiert; denn er leitete konsequent alle an die Besteuerung gestellten Forderungen aus dem Interesse des Staates selbst ab. ${ }^{576} \mathrm{Im}$ Gegensatz zur Finanzgesetzgebung der absoluten Monarchie ergaben sich für Stein - abgesehen von den bekannten „Steuerprincipien“ - drei Prinzipien der verfassungsmäßigen Finanzgesetzgebung: das Prinzip der Gesetzmäßigkeit jeder Anforderung des Staats an den Einzelnen, das Prinzip der Allgemeinheit der Leistungspflicht und das Prinzip der Gleichheit in der Leistung. ${ }^{57}$

Da der Staat auf Dauer eingerichtet sei und seine Bedürfnisse auch dauernd gegeben seien, müsse die Steuer so eingerichtet sein, dass sie dauernd gezahlt werden könne; die Steuer war daher ihrem obersten Prinzip nach eine Steuer vom Reineinkommen. Denn jede unberechtigte Einnahme des Staats bzw. jede Ausgabe, welche nicht für die Bedingungen der gesamten Volksentwicklung geschehe, müsse „streng logisch zur Armuth des Volkes führen“. ${ }^{578}$

In den beiden letzten Auflagen des Lehrbuches der Finanzwissenschaften zeigt sich, in welche Richtung sich die Stein'sche Finanzwissenschaft entwickelte. Unter dem Einfluss der neueren Literatur berücksichtigte er zunehmend die sozialen Forderungen; denn das Prinzip der sozialen Steuerbefreiung bestehe darin,

daß derjenige kein Steuersubject sein soll, dessen Einkommen zu gering ist, um noch eine kapitalbildende Kraft zu besitzen, und daß daher das Steuersubject erst bei demjenigen Einkommen anfängt, das groß genug ist, um bei verständiger Wirthschaft noch ein Kapital zu bilden. Es ist die Steuerbefreiung der nichtbesitzenden oder kapitallosen Arbeit. ${ }^{579}$

Einen völlig entgegengesetzten Standpunkt als von Stein vertrat 1866 Karl Bernhard Arwed Emminghaus, der als Klassisch-Liberaler den Staatsbedarf als unwirt-

575 Stein, Lorenz von: Lehrbuch der Finanzwissenschaft, Erster Theil, S. $53 \mathrm{f}$.

576 Ebd., S. $29 \mathrm{f}$.

577 Ebd., S. 193.

578 Ebd., S. 179.

579 Stein, Lorenz von: Lehrbuch der Finanzwissenschaft, Zweiter Theil, Die Finanzverwaltung Europas. Mit specieller Vergleichung Englands, Frankreichs, Deutschlands, Oesterreichs, Italien, Rußlands und anderer Länder. Erste Abteilung, Der Staatshaushalt, die Staatsausgaben, die wirtschaftlichen Staatseinnahmen und der allgemeine Theil der Steuerlehre, 5. Aufl., Leipzig 1885, ND Hildesheim/New York 1975, S. 409. 
schaftlich bezeichnete. Es sei keine wirtschaftliche, sondern eine politische und ethische Frage, wie viel Interesse der Einzelne an der Staatsordnung habe. ${ }^{580}$ Als überzeugter Liberaler sah er das unaufhaltsame Fortschreiten der Handelsfreiheit voraus, weshalb ihm eine einzige allgemeine Einkommensteuer als die Steuer der Zukunft erschien. Da im gleichen Maße, in dem die Zölle und die inneren Verbrauchsteuern abgeschafft würden, die direkten Steuern zunähmen, könne keine andere Steuer sich an Einfachheit und Gerechtigkeit mit dem Ideal einer einzigen Einkommensteuer vergleichen. ${ }^{581}$

Emminghaus wollte mit der Einkommensteuer eine erzieherische Funktion verbinden: Nicht das individuelle effektive Einkommen, sondern das Soll-Einkommen, das „der Steuerpflichtige unter Berücksichtigung seiner gesamten ökonomischen Lage und bei beständiger Ausbeutung derselben erzielen müßte" ${ }^{\text {"582 }}$, wollte er besteuern. Damit umging er die steuertechnischen Bedenken, die viele Autoren gegenüber der Feststellung des Reineinkommens erhoben hatten. ${ }^{583}$

Bis in die Sechzigerjahre des 19. Jahrhunderts hinein wurden die individualistische Staats- und Gesellschaftslehre und das Prinzip der Leistung und Gegenleistung (Äquivalenztheorie) nicht aus ihrer herrschenden Stellung verdrängt. Anfang der Sechzigerjahre entwickelte sich jedoch in der deutschen Literatur eine prinzipielle Gegnerschaft zu der individualistischen Auffassung; überhaupt begann eine neue Periode, die sich von allen vorausgehenden Perioden unterschied. ${ }^{584}$

\subsubsection{Das „ethische Steuerprinzip“ von Schäffle und Schmoller}

Den Anstoß zu einer fundamentalen Veränderung in der gesamten Auffassung des Steuerwesens gaben zwei berühmte Abhandlungen von Albert Schäffle ${ }^{585}$ und Gustav

580 Meyer, Robert: Die Prinzipien der gerechten Besteuerung, S. 118.

581 Emminghaus, Karl Bernhard Arwed: Einkommensteuer, in: Rentsch, Hermann (Hrsg.): Handwörterbuch der Volkswirtschaftslehre, Leipzig 1866, S. 246-253.

582 Mann, Fritz Karl: Steuerpolitische Ideale, S. 255.

583 Vgl. hierzu die Ausführungen in den Kapiteln 6.1.2.3 sowie 7.2.3.

584 Wilke, Gustav: Die Entwicklung der Theorie, S. 65; Meyer, Robert: Die Prinzipien der gerechten Besteuerung, S. 119.

585 Schäffle, Albert Eberhard Friedrich: Mensch und Gut in der Volkswirtschaft oder der ethisch-anthropologische und der chrematistische Standpunkt in der Nationalökonomie, mit besonderer Rücksicht auf die Grundprincipien der Steuerlehre, in: Deutsche Vierteljahresschrift 24.4. (1861), S.232-307. 
Schmoller ${ }^{586}$ aus den Jahren 1861 und 1863. Im Gegensatz zur herrschenden Theorie, welche in dem Einkommen oder Reineinkommen ein selbstständiges Steuerobjekt sah, stellte Schäffle die Steuerpflicht des Menschen, die Person des Staatsbürgers, in den Mittelpunkt der Wirtschafts- und Steuerlehre. Er wies nach, dass ein Einkommen getrennt von der wirtschaftenden Person nicht denkbar ist, dass es nur durch den Bezug auf den Menschen als solches existiert. Nicht mehr der künstlich konstruierte, abstrakte, sondern der wirklich konkrete, von ethischen Motiven bewegte Mensch mit all seinen Bedürfnissen wurde jetzt der wissenschaftlichen Betrachtung zugrunde gelegt. ${ }^{587}$ Seine wirtschaftliche Leistungsfähigkeit wurde nicht mehr nach dem abstrakten, auf die Verhältnisse der Produktion berechneten Begriff des reinen Einkommens gewertet, sondern kam in den die wirtschaftliche Kraft des Menschen doch erst tatsächlich bestimmenden Bedürfnissen zum Ausdruck.

Schon Schäffle verlangte eine Besteuerung der gesamten "qualificirten" Vermögenspersönlichkeit und bestimmte das Einkommen als die Summe wirtschaftlicher Güter, die ein Mensch in einer gewissen Zeit zur Befriedigung seiner Bedürfnisse ohne Schmälerung seines Vermögens verwenden könne. ${ }^{588}$ Auch Schmoller teilte diesen Einkommensbegriff und charakterisierte das Verhältnis des Einzelnen zum Staat als „Unterthanschaft"; denn die Steuerpflicht beruhe auf der Bürgerpflicht schlechthin. ${ }^{589}$

In seinem 1880 erschienenen Buch „Die Grundsätze der Steuerpolitik und die schwebenden Finanzfragen Deutschlands und Oesterreichs ${ }^{\text {“590 }}$ sowie in dem 1895/97 veröffentlichen zweibändigem Werk „Die Steuern ${ }^{\text {“591 }}$ ging Schäffle unter anderem auf die direkte und indirekte Besteuerung ein, da ihm eine notwendige systematische Einheit des Steuersystems nur in der Kombination von direkten und indirekten

586 Schmoller, Gustav: Die Lehre vom Einkommen in ihrem Zusammenhang mit den Grundprincipien der Steuerlehre, in: Zeitschrift für die gesamte Staatswissenschaft 19 (1863), S. 1-86, S. 52: „Das Einkommen ist kein Produkt eines beliebigen wirthschaftlichen Rechnungsexempels, sondern ein lebendiges Ganzes, wie es aus dem Begriffe der Persönlichkeit in ihrem Zusammenhang mit der Bedürfnissbefriedigung hervorgeht."

587 Meyer, Robert: Die Prinzipien der gerechten Besteuerung, S. $126 \mathrm{f}$.

588 Ebd., S. 127; Wilke, Gustav: Die Entwicklung der Theorie, S. 80.

589 Wilke, Gustav: Die Entwicklung der Theorie, S. 80.

590 Schäffle, Albert Eberhard Friedrich: Die Grundsätze der Steuerpolitik und die schwebenden Finanzfragen Deutschlands und Oesterreichs, Tübingen 1880.

591 Schäffle, Albert Eberhard Friedrich: Die Steuern, Allgemeiner Teil, in: Frankenstein, Kuno (Hrsg.): Hand- und Lehrbuch der Staatswissenschaften, Zweite Abteilung: Finanzwissenschaft, Bd. 2, Leipzig 1895, S. 1-420. 
Steuern möglich erschien; denn beide setzten einander voraus und ergänzten sich gegenseitig, indem die eine dasjenige erfasse, was der anderen entgehe. ${ }^{592}$

Das System der direkten Steuern wollte Schäffle aus fünf Partialeinkommensteuern zusammensetzen: aus der Grundsteuer, die er aus den wirklichen persönlichen Reineinkünften aus dem Grundbesitz ermitteln wollte, aus der Gebäudesteuer, die er zu einer Steuer auf die wirklichen reinen Einkünfte aus dem Gebäude fortbilden wollte, aus der Steuer auf Handel und Gewerbe, die er in eine feste Steuer in Abhängigkeit der verschiedenen Gewerbearten in den verschiedenen Ortsgrößen nach dem Unterschied ihrer mittleren Einträglichkeit und in eine bewegliche Steuer auf die nach objektiven Merkmalen festzustellende besondere Einträglichkeit des einzelnen Geschäfts geteilt wissen wollte, aus der Rentensteuer und aus der Steuer vom Dienst- und Berufseinkommen. ${ }^{593}$ Diese kombinierten Partialeinkommensteuern sollten je nach Staatsbedarf jährlich erhöht oder vermindert werden. Die indirekten Konsumsteuern einschließlich der Zölle begrenzte Schäffle vorwiegend auf den Verund Gebrauch an sich entbehrlicher, aber allgemein begehrter Gegenstände unter ausdrücklicher Ausschließung von Erzeugnissen des notwendigen Lebensbedarfs. ${ }^{594}$

Beide Autoren, sowohl Schäffle als auch Schmoller, haben mit ihren Abhandlungen die Grundlagen für die weitere Entwicklung geschaffen, ohne dass man ihre Ausführungen als abgeschlossene, fertige Theorie bezeichnen kann. ${ }^{595}$ Das von ihnen aufgestellte „ethische“ Steuerprinzip, wonach jeder nach seiner gesamten ökonomisch-politischen Leistungsfähigkeit das Wohl der Gesamtheit zu fördern verpflichtet sei, ${ }^{596}$ wurde in der Folgezeit von einer Gruppe von Autoren aufgegriffen, die sich mit der Reform der direkten Steuern beschäftigten. Zuvor lebte jedoch das Ideal des absolutistischen Zeitalters, alle Steuerarten in einer Universalakzise zusammenzufassen, wieder auf. ${ }^{597}$

\subsubsection{Die Theorie der Ausgabenbesteuerung}

In der Literatur tritt eine Erscheinung zutage, die völlig im Gegensatz zur herrschenden Lehre stand: eine Theorie der Ausgabenbesteuerung, die den Verbrauch

592 Ebd., S. $80 \mathrm{ff}$.

593 Schäffle, Albert Eberhard Friedrich: Die Grundsätze der Steuerpolitik, S. 294 f., sowie Ders.: Die Steuern, S. 170 f. und S. $218 \mathrm{f}$.

594 Schäffle: Grundsätze S. 88 f.; Ders.: Die Steuern, S. 257 f. und S. 325 f.

595 Meyer, Robert: Die Prinzipien der gerechten Besteuerung, S. $129 \mathrm{f}$.

596 Ebd., S. 138.

597 Mann, Fritz Karl: Steuerpolitische Ideale, S. 257 f. 
in den Mittelpunkt stellte. Zu ihren Vertretern zählten Eduard Pfeiffer, Heinrich Maurus, Hugo Alexander Eisenhart und Leon von Bilinski. ${ }^{598}$

Eduard Pfeiffer wollte sämtliche Abgaben durch eine einzige proportionale Verbrauchsteuer ersetzen. ${ }^{599}$ Er hob hervor,

daß das Prinzip auf welchem diese Steuer fußt, als das gerechteste gelten muß, weil durch sie allein eine gleichmäßige Verteilung der Staatslasten ermöglicht wird. Denn sie allein gibt die Möglichkeit, die Leistungen für den Staat so zu vertheilen, daß jedem Einzelnen ein gleiches Opfer zugemuthet werde. ${ }^{600}$

Er begründete seine Auffassung damit, dass die Einkommensteuer zu mancherlei Ungleichheiten und Bevorzugungen führe und außerdem wie eine Strafe für den Fleißigen und Sparsamen wirke. Denn mit ihr nehme der Staat seinen Löwenanteil, sobald es jemanden durch besondere Anstrengung gelungen sei, seine wirtschaftliche Lage zu verbessern. ${ }^{601}$ Im Gegensatz dazu könne bei der Verbrauchsteuer jeder seinen Steuerbetrag, den er zu entrichten habe, falls er ihm zu hoch erscheine, so ermäßigen, wie er wolle. Auch in finanzieller Hinsicht böten sich Vorteile, denn die Kosten der Erhebung seien gering, die Erhebung leicht und sicher, und Steuerumgehungen könnten daher kaum vorkommen. ${ }^{602}$ Aus der damaligen Zeit heraus ist verständlich, dass Pfeiffer darauf verwies, dass durch die Einführung einer allgemeinen Verbrauchsteuer der Weg gegeben wäre, auf dem man zu der lang ersehnten Einheit in der Besteuerung der verschiedenen deutschen Staaten gelangen könnte. ${ }^{603}$

$\mathrm{Zu}$ einem ähnlichen Resultat gelangte Heinrich Maurus, der die Beitragsfähigkeit als Grund der Besteuerung verwarf und den durch die Staatstätigkeit gesicherten Genuss als Grundlage und Maßstab der Besteuerung anerkannte. Er hielt die Steuer für ungerecht, wenn sie dem Besteuerten mehr nehme, als der Staat ihm für Lebenszwecke, wie Sicherheit etc. gebe. Die Einkommensbesteuerung hielt er für gefährlich, da diese einerseits eine Strafe für Fleiß und Geschicklichkeit darstelle und andererseits die ersparten Einkommensteile mit belaste; er verlangte vielmehr eine allgemeine Besteuerung der Artikel des Genusses und des Luxus. Die Durchführung dachte Maurus sich in Form einer indirekten, bei den Produ-

598 Meyer, Robert: Die Prinzipien der gerechten Besteuerung, S. 130.

599 Pfeiffer, Eduard: Die Staatseinnahmen, Geschichte, Kritik und Statistik derselben, Stuttgart/ Leipzig 1866, S. 554.

600 Ebd., S. $543 \mathrm{f}$.

601 Ebd., S. 544.

602 Ebd., S. 545 f.

603 Ebd., S. 554. 
zenten erhobenen Steuer. Dazu sollte noch eine Abgabe für arbeitsfähige, aber ihre Arbeitskraft nicht benutzende Staatsbürger kommen, da jedermann verpflichtet sei, in der Gesellschaft zu arbeiten. ${ }^{604}$

Hugo Alexander Eisenhart hielt die allgemeine Einkommensteuer nur insofern für brauchbar, als sie dem unvorhergesehenen und außerordentlichen Staatsbedarf genügen sollte. Für die gewöhnlichen Verhältnisse hielt er die Schwierigkeit der Einkommensermittlung und die Lästigkeit des Eindringens in die Privatverhältnisse für zu bedeutend. An ihre Stelle sollte ein System direkter und indirekter Verbrauchsteuern treten, die jedoch die notwendigen Bedürfnisse der Steuerbürger nicht belasten dürften. ${ }^{605}$

Leon von Bilinski wollte die Luxussteuer zusammen mit den Verbrauchsteuern - neben dem Ertragsteuersystem und der allgemeinen Einkommensteuer - zur Ergänzung der noch übrig gelassenen Lücken verwenden. Mit der Luxussteuer sollte der hauptsächliche Verbrauch der Reichen besteuert werden. Er sah darin auch eine Möglichkeit, einen Teil der sozialen Frage zu lösen, da damit die Reichen verhältnismäßig höher belastet würden als die Armen und sie daher ein wichtiges und unentbehrliches Glied eines rationellen Steuersystems wäre. ${ }^{606}$

\subsubsection{Die Reform des direkten Steuerwesens}

Zahlreiche Autoren knüpften an die Theorien von Schäffle und Schmoller an und machten Vorschläge zu einer Weiterentwicklung dieser neuen Lehre. Carl Walcker ${ }^{607}$ verwarf 1869 die indirekten Steuern u. a. wegen ihres unethischen Charakters vollständig und forderte eine den einzelnen Einkommensarten angepasste Einkommensteuer. Er bekannte sich ausdrücklich zu dem von Schäffle und Schmoller aufgestellten „ethischen“ Steuerprinzip, wonach jeder nach seiner gesamten ökonomisch-politischen Leistungsfähigkeit das Wohl der Gesamtheit zu fördern verpflichtet sei. Er lehnte die von Pfeiffer verlangte Steuerfreiheit der Ersparnisse

604 Maurus, Heinrich: Die moderne Besteuerung und die Besteuerungsform vom Standpunkte des gemeinen Rechts, Heidelberg 1870, S. $120 \mathrm{ff}$.

605 Eisenhart, Hugo Alexander: Die Kunst der Besteuerung, Berlin 1868, S. 145 und S. 170 ff.

606 Bilinski, Leon von: Die Luxussteuer als Correctiv der Einkommensteuer. Finanzwissenschaftlicher Beitrag zur Lösung der socialen Frage, Leipzig 1875, S.7-133.

607 Walcker, Carl: Die Selbstverwaltung des Steuerwesens im Allgemeinen und die russische Steuerreform. Mit besonderer Berücksichtigung der Projecte der allerhöchst verordneten russischen Steuerreform-Commision. Zugleich ein Beitrag zur Kenntnis innerrussicher und baltischer Zustände und Parteien, Berlin 1869. 
ab und verwarf auch die Steuerprogression als Ungerechtigkeit gegenüber den Reichen. Auch die Erbschaftsteuer sei überhaupt eine irrationale Steuerform. ${ }^{608}$

Adolf Held sah das Besteuerungsrecht im Wesen des Staats begründet und führte 1872 aus, dass der Staat nicht dem Einzelnen nehme, was diesem kraft ursprünglichen Rechts gehöre, sondern was er notwendig brauche und worauf er ein ursprüngliches Recht habe. ${ }^{609}$ Aus diesem Grund forderte Held als erste Regel der Steuerverteilung die Allgemeinheit der Steuer, d.h., jeder, der ein Einkommen habe, solle Steuer zahlen. Als zweite Regel hob er die Gleichheit der Steuern heraus, d.h., das Einkommen, das in verschiedenen Produktionszweigen erworben werde, solle ohne Rücksicht auf die Art seiner Quelle gleichmäßig besteuert werden. Der dritte Grundsatz bestand in einer weitestgehenden Schonung des Nationalwohlstandes dergestalt, dass weder den Ärmeren die Existenz gefährdet noch den Reicheren die Möglichkeit genommen werde, Kapital anzusammeln. ${ }^{610}$

Die Einkommensteuer als einzige Steuer verwarf Held und verlangte für die unteren Klassen nur eine ganz leichte direkte Steuer und ein System indirekter Steuern auf entbehrliche Genussgegenstände. Im Interesse der Einfachheit der Steuern hielt er Unterschiede mit Rücksicht auf individuelle Verhältnisse sowie auf die Beschaffenheit des Einkommens für entbehrlich. Bei den Steuersätzen wollte er deshalb auch bei den unteren Klassen eine genaue Abschätzung des Einkommens vermeiden und nur wenige progressive Sätze vorsehen und schlug beispielhaft vier Klassen vor:

I. Einkommen bis 300 Thaler $1-2 \%$,

II. Einkommen von 300 bis 1000 Thaler 3-4\%,

III. für Personen von 1000-3000 Thaler Einkommen als auch

IV. für Personen über 3000 Thaler Einkommen wäre ein Steuersatz von $5 \%$ denkbar, der in Notzeiten bei Klasse IV. auf $10 \%$ und darüber gesteigert werden könnte. ${ }^{611}$

Held war einer der Gutachter des im Jahr 1873 gegründeten Vereins für Socialpolitik, der sich in seiner Anfangszeit mit der Personalbesteuerung beschäftigte. Fünf Gutachter äußerten sich zu den vom Verein aufgeworfenen Fragen, ob das Einkommensteuerprinzip auf alle Klassen der Gesellschaft anwendbar sei, ob die

608 Meyer, Robert: Die Prinzipien der gerechten Besteuerung, S. $137 \mathrm{f}$.

609 Held, Adolf: Die Einkommensteuer. Finanzwissenschaftliche Studien zur Reform der directen Steuern in Deutschland, Bonn 1872, S. 35.

610 Ebd., S. 121.

611 Ebd., S.215f. 
Einkommensteuer progressiv sein bzw. „fundiertes“ Einkommen stärker belasten solle, ob eine Selbsteinschätzung stattfinden solle, ob und welche indirekten Steuern bei der Heranziehung nach der persönlichen Leistungsfähigkeit entfallen könnten. Gutachter waren die Professoren Erwin Nasser, Adolf Held, Constantin Rößler und Graf Wintzigerode sowie der Sekretär der Handelskammer Leipzig Julius Gensel. ${ }^{612}$

Alle Gutachter stellten das Prinzip der Leistungsfähigkeit in den Vordergrund, waren jedoch in Einzelfragen unterschiedlicher Meinung. Gensel und Wintzingerode lehnten die Steuerfreiheit des Existenzminimums prinzipiell ab, hielten jedoch die Progression für notwendig. Gensel fügte hinzu, dass nur so die Gleichheit der Opfer erzielt werde. Außerdem wollte er das Vermögen selbstständig besteuern, da dies der wesentliche Inhalt der Forderung nach einer stärkeren Besteuerung des fundierten Einkommens sei. Rößler hingegen leitete aus dem Prinzip der Leistungsfähigkeit die Konsequenz ab, dass das Existenzminimum unbesteuert bleiben solle und individuelle Verschiedenheiten der Leistungsfähigkeit Berücksichtigung finden müssten. Die Progression lehnte er als kommunistisches Projekt ebenso wie die Vermögensteuer ab. Nasser verwarf den Maßstab der Leistungsfähigkeit, da diese durch so viele Momente beeinträchtigt würde, dass dieser Maßstab der Willkür Tür und Tor öffne. ${ }^{613}$

Diesem letzten Argument trat Friedrich Julius Neumann mit einem weiteren vom Verein für Socialpolitik eingeholten Gutachten vor der Verabschiedung des Einkommensteuergesetzes 1874 entgegen. Zunächst verwies er darauf, dass es sich bei dem Abgabewesen des Staates um eine Gemeinwirtschaft zur Erreichung höherer sittlicher Zwecke handele. Wie im Privatleben, wo es sich um die Erfüllung sittlicher Pflichten - z. B. der Erziehung der Kinder oder der Sicherung der Eltern - handele, sei die Leistungsfähigkeit ein gerechter Maßstab der Leistung, so könne sich auch die sittliche Leistung für den Staat nur nach der Leistungsfähigkeit bestimmen. Nur auf diese Weise sei es auch möglich, dass der Staat - was er im Notfall tun müsse - die ganze Kraft seiner Angehörigen in Anspruch nehmen könne. Die Abgaben auf dieser Grundlage nennt Neumann „Steuern“, die Abgaben, die auf dem Prinzip von Leistung und Gegenleistungen beruhen, „Gebühren und Beiträge“.614

Dem von vielen Seiten erhobenen Vorwurf, aus der Leistungsfähigkeit lasse sich kein rechnerischer Maßstab ableiten, begegnete Neumann mit dem Hinweis, dass sich auf vielen Gebieten, in denen Gerechtigkeit ausgeführt werden müsse, kein

612 Meyer, Robert: Die Prinzipien der gerechten Besteuerung, S. $142 \mathrm{f}$.

613 Ebd., S. $143 \mathrm{f}$.

614 Neumann, Friedrich Julius: Die progressive Einkommensteuer im Staats- und Gemeinde-Haushalt. Gutachten über Personalbesteuerung, auf Veranlassung des Vereins für Socialpolitik (Schriften des Vereins für Socialpolitik 8), Leipzig 1874, S. 58-70. 
rechnerischer Maßstab finden lasse und demnach in Zahlen entschieden werden müsse. Die Schwierigkeit der Ausführung könne die Richtigkeit des Prinzips nicht infrage stellen. Er zog aus diesem Grundsatz auch die entsprechenden Konsequenzen: progressive bzw. degressive Besteuerung, da das höhere Einkommen regelmäßig leichter entbehrlichen Ausgaben diene, ${ }^{615}$ und Freilassung der unteren Klassen von direkten Steuern. Nach Neumann litten die unteren Klassen unter den direkten Steuern ungleich empfindlicher als die höheren. Daher sei ihre direkte Besteuerung - unabhängig von der wirtschaftlichen Unzweckmäßigkeit - eine Ungerechtigkeit. ${ }^{616}$ Außerdem forderte er die Nichtberücksichtigung des Erwerbs der unselbstständigen Familienmitglieder bei der Einschätzung des Einkommens. ${ }^{617}$ Eine geringere Leistungsfähigkeit infolge von Verschuldung und Alter, Krankheit, Kinderzahl und außerordentlicher Unglücksfälle solle berücksichtigt werden, ${ }^{618}$ und die Einkommensteuer solle mit einer Vermögensteuer kombiniert werden. ${ }^{619}$

Die Forderung nach progressiver Besteuerung wurde von Friedrich Wilhelm Hans von Scheel unterstützt. Er hielt die Berücksichtigung der sozialen Verhältnisse insbesondere darum für notwendig, weil die zu seiner Zeit auftretenden und sich verschärfenden Vermögensungleichheiten die soziale und politische Freiheit gefährdeten. ${ }^{620}$ Unter Berücksichtigung der „sozialen Steuerfähigkeit“ hielt Scheel einen Normalsteuersatz, der die standesmäßige Lebenshaltung in keiner Weise beeinträchtigt, für geboten. Er hielt es für selbstverständlich, „dass auch die höchste Stufe der Progressivsteuer einen genügenden Spielraum nicht nur für die Lebenshaltung, sondern auch für den Erwerbstrieb lassen [...] muss. “621 Weiterhin vertrat er den Standpunkt, dass die Verringerung des Steuersatzes bei abnehmendem Einkommen (Degression) nicht zur völligen Steuerfreiheit führen dürfe, da die Steuerpflicht sich unmittelbar aus der Staatsangehörigkeit ergebe und niemand ein Recht auf Steuerfreiheit habe. ${ }^{622}$ Eine stärkere Belastung des fundierten Einkommens solle insbesondere bei Erbübergang erreicht werden, bei dem das Prinzip der Progression auch greifen solle, um den sozialen Frieden herzustellen. ${ }^{623}$

615 Ebd., S. $106 \mathrm{ff}$.

616 Ebd., S. $154 \mathrm{ff}$.

617 Ebd., S. $171 \mathrm{ff}$.

618 Ebd., S. $176 \mathrm{f}$.

619 Ebd., S. $185 \mathrm{ff}$.

620 Scheel, Friedrich Wilhelm Hans von: Die progressive Besteurung, in: Zeitschrift für die gesammte Staatswissenschaft 31 (1875), S. 289-303, S. 290 f.

621 Ebd., S. 296.

622 Ebd., S. 291.

623 Ebd., S. 302. 
Georg Hirth befürwortete die Progression aus doppeltem Grund: Erstens verwies er darauf, dass der Staat ein weit höheres Anrecht auf ein relativ müheloses, durch ererbte Kapitalien, Spekulationen etc. erzieltes Einkommen habe als auf dasjenige, was als Frucht harter Arbeit anzusehen sei. Er hielt es für vollkommen gerecht, wenn der Staat seinen Anteil an dem Gewinn nach dem Interesse bemesse, welches die Bürger an der Aufrechterhaltung staatlicher Ordnung hatten; dieses Interesse des einzelnen Besitzenden wachse nach seiner Ansicht progressiv. ${ }^{624}$ Zweitens schien es ihm weise und gerecht zu sein, das Einkommen und Vermögen der Bürger dergestalt mit wachsenden Prozentsätzen zu besteuern, dass dem Wohlhabenden immer noch Mittel zum Luxus und zur Verschwendung in ausreichendem Maß zur Verfügung blieben. Die direkte progressive Einkommensteuer würde die Wohlhabenden nach ihrer wirklichen Steuerkraft zu den Staatslasten heranziehen und die Überbürdung der Unbemittelten mit Steuern beseitigen. ${ }^{625}$

Ein gemäßigter Anhänger der progressiven Einkommensteuer war Karl Burkhart, ${ }^{626}$ der die Degression der Progression vorzog, da nicht die Mehrbelastung der höheren, sondern die Minderbelastung der niedrigen Einkommen das Wesentliche sei. ${ }^{627}$

In den Schriften des Vereins für Socialpolitik wurden im Jahr 1877 zehn Gutachten publiziert, die im Wesentlichen denselben Standpunkt wie Neumann einnahmen. Die Autoren Bruch, Wolf, Meier, Reitzenstein, Heitz und Samter waren sich über die Zugrundelegung des Leistungsprinzips einig. Bis auf Heitz - der die Progression für den Staat nicht, dagegen für die Gemeinden zulassen wollte - akzeptierten alle die progressive Besteuerung und eine stärkere Besteuerung des fundierten Einkommens bzw. eine Kombination der Einkommensteuer mit einer Vermögensteuer. ${ }^{628}$

624 Hirth, Georg: Über Culturpolizei und Rechtsgleichheit. Zur Lösung der socialen Frage, in: Ders. (Hrsg.): Annalen des Deutschen Reiches für Gesetzgebung, Verwaltung und Statistik, Leipzig 1873, S. $848 \mathrm{f}$.

625 Ebd., S. $851 \mathrm{f}$.

626 Burkhart, Karl: Die bestehenden Einkommensteuern. Eine vergleichende Darstellung, im Auftrage des deutschen Steuer-Reformvereins bearbeitet, Leipzig 1875.

627 Meyer, Robert: Die Prinzipien der gerechten Besteuerung, S. 149.

628 Ebd., S. 150. 


\subsubsection{Der Einfluss der sozialpolitischen Bewegungen auf steuerpolitische Ideale}

\subsubsection{Der proletarische Sozialismus}

Nach dem Ende der Märzrevolution von 1848 und der Aufhebung der Pressezensur brachte der junge Karl Marx als Redakteur der Neuen Rheinischen Zeitung seine Ablehnung des Staates und der politischen Institutionen auf den Punkt: „Von dem heutigen Tag an sind die Steuern aufgehoben!!! Die Steuerzahlung ist Hochverrat, die Steuerverweigerung erste Pflicht des Bürgers!"“229 Diese radikale Steuerverweigerungskampagne hielt Marx und Friedrich Engels jedoch nicht davon ab, ein praktisches Steuerprogramm zu entwerfen. Im „Kommunistischen Manifest“ von 1848 wurde eine „starke Progressionssteuer“ genannt, die allmählich bis zur Enteignung gesteigert werden sollte, um „der Bourgeoisie nach und nach alles Kapital zu entreißen. “630 Unabhängig davon konzentrierten sich Marx und Engels auf die wissenschaftliche Kritik der indirekten Steuern, was Ferdinand Lassalle in seiner Kammergerichtsrede von 1863 zu heftigen Anklagen gegen die indirekten Steuern führte. ${ }^{631}$ Lassalle trug alle wirkungsvollen Beweisgründe zusammen, um die verheerende Wirkung der indirekten Steuern zu beweisen. Er bediente sich dabei eines Kunstgriffes, denn er zählte auch die Grund- und die Gewerbesteuer zu den indirekten Steuern und konnte damit behaupten, dass der Hauptteil der indirekten Steuern nicht nur von den ärmeren Klassen getragen werde, sondern jemanden umso stärker treffe, je ärmer er sei. ${ }^{632}$

Das neue Ideal fand in den Forderungen des Eisenacher Programms der Sozialdemokratischen Arbeiterpartei 1869 unter III. Nr. 9 seinen Niederschlag: „Abschaffung aller indirekten Steuern und Einführung einer einzigen Einkommensteuer und Erbschaftsteuer" ${ }^{\text {" }}{ }^{33}$ Nachdem im Gothaer Programm von 1875 die Erbschaftsteuer

629 Marx, Karl: An das preußische Volk, Beilage der Neuen Rheinischen Zeitung Nr. 145 vom 17.11.1848.

630 Marx, Karl/Engels, Friedrich: Das kommunistische Manifest, (1847/48), 8. Aufl., Berlin 1918, S. $44 \mathrm{f}$.

631 Lassalle, Ferdinand: Die indirekte Steuer und die Lage der arbeitenden Klassen. Eine Verteidigungsrede vor dem königlichen Kammergericht zu Berlin gegen die Anklage, die besitzlosen Klassen zum Haß und zur Verachtung gegen die Besitzenden öffentlich angereizt zu haben, in: Ders.: Gesammelte Reden und Schriften, Bd. 2, Berlin 1893, S. 241-389.

632 Ebd., S.302.

633 Sozialdemokratische Arbeiterpartei: Das Eisenacher Programm der Sozialdemokratischen Arbeiterpartei vom 08.08.1869, in: Treue, Wolfgang (Hrsg.): Deutsche Parteiprogramme seit 1861, Quellensammlung zur Kulturgeschichte, Bd. 3, 4. Aufl., Zürich/ 
entfernt und als Ersatz der indirekten Steuern nur eine einzige progressive Einkommensteuer dienen sollte, forderte die Sozialdemokratische Partei Deutschlands im Erfurter Programm von 1891 unter Nr. 9 eine stufenweise steigende Einkommens-, Vermögens- und Erbschaftsteuer, und außerdem die „Abschaffung aller indirekten Steuern, Zölle und sonstigen wirtschaftspolitischen Maßnahmen, welche die Interessen der Allgemeinheit den Interessen einer bevorzugten Minderheit opfern. "“634

\subsubsection{Die bürgerliche Sozialreform}

In der zweiten Hälfte des 19. Jahrhunderts eskalierten die sozialen Spannungen in der Bevölkerung. Der freie Wettbewerb und die industrielle Revolution hatten eine herausragende Entwicklung der Wirtschaftskräfte verursacht, aber gleichzeitig zu sozialen Spannungen geführt.

Sowohl die Sozialdemokratische Arbeiterpartei als auch die christlich-soziale Arbeiterpartei traten für eine grundlegende Steuerpolitik ein, in der die sozialen Belange eine gewichtige Rolle spielen sollten ${ }^{635}$ Sozialpolitik sollte fortan nicht nur im Geben, sondern auch im Nehmen vollzogen werden. Dabei bewegten sich die Steuerreformen in dreifacher Richtung: ${ }^{66}$ Die direkten Steuern wurden erstens mit Vergünstigungen für die ärmeren Volksschichten ausgestattet. Ein Teil der steuerlichen Lasten wurde zweitens von den indirekten auf die direkten Steuern übertragen, um die Wirtschaftsschwachen zu entlasten. Und drittens sollte die Steuer nicht nur ein fiskalisches Hilfsmittel sein, sondern auch als Regulator der Wohlstandsverhältnisse eingesetzt werden und als Hebel der Gesellschaftsreform dienen. Das dritte Verfahren bedeutete einen offenen Bruch mit der naturrechtlich-liberalen Überlieferung.

Berlin/Frankfurt am Main, S.69-71; https://www.marxists.org/deutsch/geschichte/ deutsch/spd/1869/eisenach.htm (letzter Zugriff: 28.02.2017).

634 Sozialdemokratische Partei Deutschlands: Das Erfurter Programm der Sozialdemokratischen Partei Deutschlands vom 21.10.1891, in: Mommsen, Wilhelm (Hrsg.): Deutsches Handbuch der Politik, Bd. 1: Deutsche Parteiprogramme, 2. Aufl., München 1960, S. 349-353. www.marxists.org/deutsch/geschichte/ deutsch/spd/1891/erfurt.htm. (letzter Zugriff: 28.02.2017).

635 Sozialdemokratische Arbeiterpartei: Das Eisenacher Programm, S. 69 f.; Christlich-Soziale Arbeiterpartei: Das Programm der Christlich-Sozialen Arbeiterpartei vom Januar 1878, in: Treue, Wolfgang (Hrsg.): Deutsche Parteiprogramme seit 1861 (Quellensammlung zur Kulturgeschichte 3), 4. Aufl., Zürich/Berlin/Frankfurt am Main 1968, S.77-79.

636 Mann, Fritz Karl: Steuerpolitische Ideale, S. 305 f. 


\subsubsection{Die Besteuerung als Instrument einer gezielten Einkommens- und Vermögensumverteilung}

\subsubsection{Die Wohlstandskorrektur als gesellschaftspolitisches Ziel}

Adolph Wagner veranlassten die sozialen Spannungen dazu, der Besteuerung neben der rein finanziellen auch eine sozialpolitische Aufgabe zuzuschreiben, um bei der im freien Wettbewerb entstandenen Einkommens- und Vermögensverteilung korrigierend einzugreifen. ${ }^{637}$ Wie kaum ein anderer zeitgenössischer Nationalökonom forderte er zur praktischen Verwirklichung der beiden Postulate der Gerechtigkeit - Allgemeinheit und Gleichmäßigkeit - auf Veränderungen der Arbeitsteilung und der Technik der Produktion Rücksicht zu nehmen: „Die Besteuerung hat sich demgemäss immer zu verändern, um gerecht zu werden oder zu bleiben." ${ }^{\text {"638 }}$

Für Wagner war der Steuerbegriff ein doppelter, je nach dem möglichen Doppelzweck der Steuer. ${ }^{639}$ Zum einen waren nach ihm Steuern im rein finanziellen Sinn diejenigen Auflagen und Abgaben, welche als Zwangsbeiträge von Einzelwirtschaften zur Deckung der allgemeinen öffentlichen Ausgaben in einseitig bestimmter Weise und Höhe nach allgemeinen Grundsätzen und Maßstäben eingefordert wurden. Zum anderen waren Steuern im „socialpolitischen“ Sinn diejenigen Auflagen und Abgaben, welche zugleich oder allein dem Zwecke dienten, in die Verteilung des Volkseinkommens und Volksvermögens und eventuell weiter in die Verwendung des Einzeleinkommens und Vermögens regulierend und verändernd einzugreifen. ${ }^{640}$

Wagner begründete seinen doppelten Steuerbegriff damit, dass dieser als Realbegriff allen geschichtlichen Tatsachen der Besteuerung gerecht werden und auf alle Arten und Formen der Steuern passen müsse. ${ }^{641}$ Der Grund der Steuer war nach Wagner vorhanden, wenn eine der beiden oder beide Zwecke der Steuer vorlagen, d.h., wenn die Steuer als „rein finanzielle“ Maßregel zur Deckung des Finanzbedarfs oder als „socialpolitische“ Maßregel zur Regulierung der bestehenden Verteilung

637 Wagner, Adolph: Finanzwissenschaft 2. Teil, S. 383.

638 Ebd., S. 387.

639 Nach Schulz ist Wagners Staatsauffassung für dessen Finanzwissenschaft von fundamentaler Bedeutung: „Indem Wagner den Machtstaat um die Funktion von Kulturund Wohlfahrtsausgaben erweitert, bewirkt er die Bildung einer eigenen staatlichen Finanzwirtschaftslehre. Der, Sinn' der Besteuerung ist bei Wagner dann nicht mehr rein fiskalisch zu bestimmen. "Vgl. Schulz, Frank: Zur Dogmengeschichte der funktionalen Finanzwirtschaftslehre. Eine literaturgeschichtliche Untersuchung zur Entwicklung der „funktional finance“ vom Merkantilismus bis zur „neuen Wirtschaftslehre“, Berlin 1987, S. 116.

640 Wagner, Adolph: Lehr- und Handbuch der politischen Oekonomie, S. 210.

641 Ebd., S. 213. 
von Einkommen und Vermögen und der Verwendung von beiden erforderlich war oder für erforderlich galt. ${ }^{642}$

Mit dieser Definition und Begründung der Steuer verwies Wagner darauf, dass bis über die Mitte des 19. Jahrhunderts hinaus vielfach andere Begründungen für die Steuer vertreten worden waren. So wurde die Steuerpflicht auf die Vorteile im Staatsverband und auf den Schutz des Staates für Person und Eigentum zurückgeführt; es wurden eine „Genusstheorie“ und eine „Assekuranztheorie“ zur Begründung von Besteuerungsrecht und Steuerpflicht gebildet und dabei auch die Steuer als eine Art „Tausch“ oder als eine Art „Preis“ aufgefasst: ein „Tausch“ zwischen der Steuer als Leistung und der dem Einzelnen zugutekommenden öffentlichen Leistungen als Gegenleistung und der Steuer als „Preis“, welcher für den Genuss dieser Leistungen bezahlt wird. Wagner rügte diese Theorien, da diese übersähen, dass „Genüsse“, „Vortheile“, für den Einzelnen gar nicht immer vorhanden seien und eine Steuerverteilung an der Durchführung scheiterte, da diese „Vortheile“ sich jeder Schätzung entzögen. Wagner bezeichnete diese daher als „Verallgemeinerung des Gebührenprincips".643

Die Frage, was gerechte Besteuerung ist und wie man weitgehend in die entstandenen Einkommens- und Vermögensverhältnisse korrigierend eingreifen könne, hat Wagner wie folgt beantwortet: Steuergerechtigkeit ist nichts Absolutes, sondern etwas zeitlich und örtlich Relatives, das durch die Kultur des Volkes bedingt ist. So standen z.B. die ehemaligen ständischen Steuerfreiheiten und Privilegien im Einklang mit den gesamten Rechtsverhältnissen der damaligen Zeit. ${ }^{644} \mathrm{Da}$ die Gerechtigkeitsidee und die Rechtsanschauungen über die Steuerverteilung einem ständigen Wechsel unterworfen sind, kann es nach Wagner keinen endgültigen Abschluss dieser Entwicklung geben. ${ }^{65}$ Das Urteil darüber, was "gerechte“ Besteuerung ist, wird wesentlich an der Beurteilung der bestehenden Einkommens- und Vermögensverteilung gemessen. Derjenige, der die bestehende Einkommens- und Vermögensverteilung als gegebene Tatsache hinnimmt, wird den Zweck der Besteuerung in der Deckung des Finanzbedarfs sehen und einen „socialpolitischen“ Zweck der Besteuerung zurückweisen. Entsprechend wird die „Allgemeinheit“ wörtlich genommen, und die „Gleichmäßigkeit“ wird als „gleiche Verhältnismäßigkeit der Steuer zum Einkommen“ aufgefasst, mit der Folge, dass eine „proportionale“ Besteuerung akzeptiert und eine „progressive“ Besteuerung abgewiesen wird.

642 Ebd., S. 216.

643 Ebd., S. $217 \mathrm{f}$.

644 Ebd., S. $379 \mathrm{f}$.

645 Ebd., S. $475 \mathrm{f}$. 
Diese, in der individualistischen Zeitepoche unterstellte Prämisse wurde jedoch von Wagner nicht als richtig anerkannt. Ein solches „noli me tangere“ war für ihn in dieser prinzipiellen Allgemeinheit unrichtig. Neben der rein finanziellen Betrachtung müsse noch der sozialpolitische Bezug hergestellt werden, denn dieser greife korrigierend in die entstandene Einkommens- und Vermögensverteilung ein. Die Konsequenz hieraus sei, dass die Allgemeinheit nicht wörtlich genommen werden dürfe, sondern es gerechtfertigt sei, dass die „kleinen Leute“ ökonomisch entlastet und relativ geringer als die Wohlhabenderen besteuert würden. Die Gleichmäßigkeit verstand er damit als „Besteuerung möglichst im Verhältniss zu der in stärkerer Progression als das absolute Einkommen (und Vermögen) steigenden wirthschaftlichen Leistungsfähigkeit“. Damit ergab sich die prinzipielle Forderung nach der progressiven Besteuerung des höheren Einkommens, ferner einer höheren Besteuerung des fundierten Einkommens als des Arbeitseinkommens. Ein weiteres Streben nach Gerechtigkeit sah er in ergänzenden Luxussteuern und der Einführung von solchen Steuern, mittelst derer die Extrabesteuerung von zufälligen, „unverdienten“, aus „Conjuncturengewinnen“ etc. herrührenden Erwerben durchgeführt werden sollten. ${ }^{646}$

Die fortschreitende Entwicklung der Arbeitsteilung und in der Produktionstechnik erfordere eine ständige Veränderung der Besteuerung, um gerecht zu bleiben. Je einfacher die Wirtschaftsverhältnisse seien, desto einfacher könne auch die Besteuerung sein, ohne gegen die Grundsätze Allgemeinheit und Gleichmäßigkeit wesentlich zu verstoßen. Mit der Auflösung der alten Wirtschaftsverhältnisse und der Entwicklung der Produktionstechnik sei alles anders geworden; unverändert bleibende, einfache Steuern würden nun immer ungerechter. Neben der einfachen Grundsteuer sei eine Reihe von direkten und indirekten Steuern notwendig, um das Einkommen anderer Klassen als der Grundeigentümer und Bauern zu treffen.

Das verlangt auch die Gerechtigkeit, denn jetzt zersplittert sich das Nationaleinkommen in immer mehr verschiedenartige Einzeleinkommen, es differenzirt sich qualitativ und quantitativ stärker, und dem muss sich die Besteuerung durch entsprechende Specialisirung anpassen. ${ }^{647}$

Je weiter diese ökonomische und technische Entwicklung fortschreite, desto mannigfaltiger und wechselnder müsse die Besteuerung werden, um die beiden Postulate der Gerechtigkeit zu erfüllen. ${ }^{648}$

646 Ebd., S. 385.

647 Ebd., S. 388.

648 Ebd., S. 388. 
Die von Wagner vertretenen sozialpolitischen Besteuerungsideale wurden auch von Friedrich Wilhelm Hans von Scheel und dem deutschen Publizisten Constantin Frantz unterstützt, die für eine Regulierung der Wohlstandsverhältnisse plädierten. Von Scheel sprach dem Staat die Aufgabe zu, die vorhandenen sozialen Ungleichheiten durch eine gezielte Steuerpolitik zu verringern. ${ }^{649}$

Constantin Frantz erkannte ausdrücklich die freie Marktwirtschaft an, sah jedoch im Steuerwesen eine besondere ethische und sozialpolitische Funktion, die dem Staat die Möglichkeit gebe, Ungerechtigkeiten in der Verteilung von Einkommen und Vermögen abzumildern. Frantz ging so weit, sowohl bei der Einkommensteuer als auch bei der Erbschaftsteuer eine Progression zu empfehlen, die bis ins Unbestimmte ging. ${ }^{650}$

Der überwiegende Teil der zeitgenössischen Wissenschaftler - wie Friedrich Julius Neumann, Karl Friedrich von Schall, Wilhelm Vocke und Albert Schäffle - stand der Umverteilung von Einkommen und Vermögen ablehnend gegenüber. Als wesentliche Momente dieser ablehnenden Meinungen wurde von ihnen die mangelnde Vereinbarkeit mit dem Leistungsprinzip und die Gefahr einer nicht mehr zu kontrollierenden Willkür genannt. ${ }^{651}$

Für Robert Meyer war die Idee der sozialen Reform aus den geschichtlichen Umständen heraus entstanden, doch war sie nicht der logische Grund, nicht die notwendige Voraussetzung der Anerkennung dieses Prinzips. Er führte aus, dass die Anhänger der sozialpolitischen Reform sich darüber einig seien, dass durch eine solche Einrichtung die Besteuerung nur ein kleiner Teil der als sozialpolitisch notwendig anerkannten Ausgleichung erreicht werden könne. ${ }^{652}$

Unabhängig davon ergab sich, dass die Anerkennung der Notwendigkeit und Gerechtigkeit einer sozialpolitischen Funktion der Besteuerung nicht im Wi-

649 Scheel, Friedrich Wilhelm Hans von: Die progressive Besteurung, S. $289 \mathrm{ff}$.

650 „Wir wollen die Progression buchstäblich genommen wissen, so daß mit dem steigenden Einkommen auch fortwährend der Steuerfuß wächst, in indefinitum“; vgl. Frantz, Constantin: Die sociale Steuerreform als die conditio sine qua non, wenn der socialen Revolution vorgebeugt werden soll, Mainz 1881, ND Aalen 1972, Vorwort, S. 45-51 und S. 69.

651 „Ihr die Pflicht zuzuschreiben, sociale Uebelstände zu beseitigen, heißt jedenfalls durchaus Fremdartiges in sie hineinzutragen“; vgl. Neumann, Friedrich Julius: Die progressive Einkommensteuer, S. 99; Schall, Karl Friedrich von: Allgemeine Steuerlehre, in: Schönberg, Gustav von (Hrsg.): Handbuch der Politischen Oekonomie, Finanzwissenschaft und Verwaltungslehre Bd. 3, 1. Halbband, 4. Aufl., Tübingen 1897, S. 151-246, S. 151; Vocke, Wilhelm: Die Abgaben, Auflagen und die Steuer vom Standpunkte der Geschichte und der Sittlichkeit, Stuttgart 1887, ND Frankfurt am Main 1970, S. 42 f. Zur Auffassung von Albert Schäffle vgl. die Ausführungen in Kapitel 7.1.3.

652 Meyer, Robert: Die Prinzipien der gerechten Besteuerung, S. $406 \mathrm{f}$. 
derspruch stünden zu der Anerkennung des Prinzips der Besteuerung nach der Leistungsfähigkeit. Die Prinzipien der Gerechtigkeit gäben dem finanziellen wie dem sozialpolitischen Zweck der Besteuerung gegenüber die Grenze an, innerhalb derer die Durchführung des staatlichen Interesses gestattet sei. ${ }^{653}$ So seien z. B. die Steuerfreiheit oder die Steuerbegünstigung gewisser, aus sozialpolitischen Gründen zu fordernder Maßnahmen Ausfluss dieser Prinzipien der Gerechtigkeit.

Zeitgenössische Wissenschaftler sahen in den verteilungspolitischen Zielen der von Wagner empfohlenen Steuerpolitik eine große Gefahr, da eine Umverteilung von Einkommen und Vermögen regelmäßig zur Folge hätte, dass der unternehmerische Antrieb gehemmt würde und damit der Volkswirtschaft erhebliche wertschöpfende Maßnahmen verloren gingen. Viele Wissenschaftler hatten nichts gegen eine Verwendung von Steuergeldern für die Durchführung bestimmter sozialpolitischer Maßnahmen, trotzdem konnten sie sich mit einer außerfiskalischen Besteuerungspolitik nicht anfreunden. ${ }^{65}$

Obwohl Wagners Lehre von der sozialen Funktion der Besteuerung von der Wissenschaft und Praxis überwiegend abgelehnt worden war, ${ }^{655}$ drang sie doch schrittweise in die Steuerpolitik aller fortgeschrittenen Länder ein. Auch im Schrifttum wurde die Notwendigkeit sozialreformerischer Steuerwirkungen immer weniger bestritten. ${ }^{656}$ In der deutschen Finanzpolitik fand eine Neuorientierung statt; denn eine Steuerpolitik, die der Gesellschaftsreform dienen sollte, konnte nicht mehr ausschließlich finanzwirtschaftliche und volkswirtschaftliche Ziele verfolgen, sondern benötigte ein breiteres Fundament. Immer mehr wurde die Steuerpolitik als Mittel zur sozialethischen Ausrichtung der Gesellschaftsverfassung betrachtet. ${ }^{657}$

\subsubsection{Die Besteuerungsgrundsätze nach Adolph Wagner}

Adolph Wagner war im deutschen Sprachraum der erste Finanzwissenschaftler, der ein umfassendes System von Besteuerungsgrundsätzen auf der Basis des von ihm

653 Ebd., S. 407.

654 Während bei Constantin Frantz, Adolf Held und Hans von Scheel noch positive Bemerkungen zu verzeichnen waren, blieb die Mehrzahl der Autoren negativ gegenüber der sozialen Funktion der Besteuerung eingestellt; vgl. Frantz, Constantin: Die sociale Steuerreform, S. 47 ff.; Held, Adolf: Die Einkommensteuer, S. 111; Scheel, Friedrich Wilhelm Hans von: Die progressive Besteurung, S. $273 \mathrm{ff}$.; Neumann, Friedrich Julius: Die progressive Einkommensteuer, S. 99; Schulz, Frank: Zur Dogmengeschichte, S. $122 \mathrm{f}$.

655 Mann, Fritz Karl: Steuerpolitische Ideale, S. 322.

656 Ebd., S. 322.

657 Ebd., S. $322 \mathrm{f}$. 
definierten Steuerrechtsbegriffs entwickelte. Ihm war klar, dass solche Grundsätze nicht absolut und unveränderlich sind, denn viele der Besteuerungspostulate sind

wesentlich zeitlich- und örtlich-relativer Art, sie hängen mit den gegebenen Cultur-, Wirthschafts- und technischen Verhältnissen, mit den jeweiligen Volksanschauungen und mit dem Zustande des Rechtsbewusstseins und des öffentlichen Rechts zusammen [...], sie sind nicht rein logische, sondern historische Kategorieen. ${ }^{658}$

Wagner wollte mit seinen Grundsätzen das Ziel vorgeben, dem man sich in der Praxis möglichst nähern sollte. Er teilte die obersten Grundsätze der Besteuerung in insgesamt vier Gruppen mit weiterer Unterteilung ein: finanzpolitische Prinzipien, volkswirtschaftliche Prinzipien, Prinzipien der Gerechtigkeit oder der gerechten Steuerverteilung und Steuerverwaltungsprinzipien.

Die finanzpolitischen Prinzipien stellte Wagner an die Spitze, namentlich auch vor die Prinzipien der Gerechtigkeit, mit denen sich die Finanzwissenschaft - neben den Steuerverwaltungsprinzipien von Adam Smith - bis dahin überwiegend beschäftigt hatte. Die volkwirtschaftlichen Steuerprinzipien beziehen sich auch auf das Problem richtiger Steuerverteilung, betreffen aber außerdem die Interessen der Gemeinschaft.

Die beiden Prinzipien der Gerechtigkeit, die Allgemeinheit und die GleichmäBigkeit der Besteuerung, waren nach Wagner zeitlich und örtlich veränderlich, nähmen jedoch im Zeitalter persönlicher Freiheit und politischer Gleichheit die Natur von Axiomen ein, die mit dem Begriff der persönlichen Freiheit und Gleichheit gegeben seien. ${ }^{69}$

\subsection{Die Allgemeinheit der Besteuerung}

Den Grundsatz der Allgemeinheit der Besteuerung ${ }^{660}$ sah Wagner darin, dass die Besteuerung eine allgemeine staatsbürgerliche Pflicht sei, welche zunächst den Personen als Staatsbürgern obliege. Bei der Durchführung des Prinzips der Allgemeinheit müsse vielfach über den Kreis von physischen Personen hinausgegangen werden, um gerecht zu sein, und z.B. auch auf juristische Personen zugegriffen werden. Andererseits seien auch Einschränkungen der Besteuerung von natürlichen Personen notwendig, teils aus politischen und wirtschaftlichen Gründen oder aus steuertechnischen Rücksichten, oft aber auch um der Gerechtigkeit willen. Insbesondere könnten sozialpolitische Gründe zu Einschränkungen der Allgemeinheit

658 Wagner, Adolph: Finanzwissenschaft 2. Teil, S. 299.

659 Ebd., S.304f.

660 Ebd., S. 389-428. 
der Besteuerung führen, so zu einer Steuerfreiheit für die unteren, nichtbesitzenden Klassen u. a. im Interesse einer ökonomischen Schonung dieser Menschen. ${ }^{661}$

An der Steuerfreiheit des sogenannten Existenzminimums lasse sich gut die Notwendigkeit die Unterscheidung der beiden Steuergesichtspunkte, des rein finanziellen und des sozialpolitischen, zeigen; denn aus Rücksicht auf die geringe ökonomische Leistungsfähigkeit dürfen die kleinen Einkommen steuerfrei sein, und es erscheint auch als gerecht, die eigentlich Armen durch die Aufhebung von Steuern auf notwendige Lebensmittel zu entlasten. ${ }^{662}$

Eine annähernde Verwirklichung der "Allgemeinheit" könne nicht mit einer einzigen, wie immer denkbaren Steuer, sondern nur durch eine Kombination verschiedener Steuern in einem einheitlichen Steuersystem erreicht werden, das sich aufgrund der Beweglichkeit der Volkswirtschaft immer wieder anpassen und verändern müsse. Zur Durchführung dieses Postulats - wie auch der Gleichmäßigkeit - ist nach Wagner eine Kombination von Ertrags-, insbesondere Realsteuern, mit Personal-, Einkommen- und Verbrauchsteuern zweckmäßig und geboten.

\subsection{Die Gleichmäßigkeit der Besteuerung}

Die Gleichmäßigkeit der Besteuerung im Sinne einer gleichmäßigen Verteilung der Steuerlast unter den einzelnen Steuerpflichtigen folge nach Wagner als Rechtsgrundsatz aus dem Prinzip der staatsbürgerlichen Freiheit und Gleichheit. Was ist und worin besteht die Gleichmäßigkeit? ${ }^{\text {63 }}$

Bei dem Prinzip der Besteuerung nach Leistung und Gegenleistung - das man auch das privatwirtschaftliche Besteuerungsprinzip nennen kann - hält man sich an die Beziehungen zwischen dem Besteuerten und dem Staat und versucht, die Besteuerung so zu verteilen, dass sich Leistung und Gegenleistung - wenn nicht nach Wertmaßstäben, so doch proportional - in gleicher Höhe bemessen. Die Bemessung erfolgt entweder am Gegenwert, den der Besteuerte an den öffentlichen Leistungen des Staates genießt - „Genusstheorie“ oder "Genussprinzip“ - oder am Kostenaufwand des Staates, den dieser verwendet, um den „Genuss von Leben und Eigentum“ des Besteuerten zu sichern - „Assekuranztheorie“ oder „Assekuranzprinzip“. Dieses privatwirtschaftliche Prinzip findet sich heute noch vielfach in dem System von Gebühren und Beiträgen.

Mit der wachsenden Ausdehnung der öffentlichen Tätigkeiten, der damit verbundenen Anerkennung der öffentlichen Leistungen des Staates und der Entwicklung des technischen, wirtschaftlichen und kulturellen Fortschritts rückte die

661 Ebd., $392 \mathrm{f}$.

662 Ebd., S. 405.

663 Ebd., S. $428 \mathrm{ff}$. 
Besteuerung nach der wirtschaftlichen Leistungsfähigkeit des Besteuerten immer mehr in den Vordergrund.

Die Begründung hierfür liegt in der Natur des Staates und in dem Verhältnis des Besteuerten zu diesem Staat. Daraus ergibt sich die Besteuerung nicht als Gegenleistung für Genüsse oder Vorteile, sondern als eine notwendige Pflicht des Besteuerten zur Erfüllung der Existenzbedingungen des Staates, dem er angehört. Der Maßstab der Steuerverteilung wird in der wirtschaftlichen Leistungsfähigkeit jedes Steuerpflichtigen als Glied einer Gemeinschaft gefunden, das gleiche verhältnismäßige „Opfer" an wirtschaftlichen Gütern zu tragen hat. Dieses Prinzip kann man das staatswirtschaftliche Steuerprinzip nennen, und man spricht auch von dem „Opferprinzip“ oder einer „Opfertheorie“.

Nach Wagner liegt die wirtschaftliche Leistungsfähigkeit einer Person in zwei „Momenten“, die einerseits den Erwerb und Besitz von Sachgütern und andererseits die Verwendung dieser Güter zur Bedürfnisbefriedigung betreffen. ${ }^{664}$ Das erste „Moment" ist der Normalfall des Erwerbs, der Einkommensbezug, der in die drei Kategorien Arbeits-, Renten- und gewerbliches Einkommen zerfällt. Das zweite "Moment" betrifft die Verschiedenheit der Lasten und Verpflichtungen, die auf dem Steuerpflichtigen liegen und die "Steuerfähigkeit“ beeinflussen. Vor allem in den Familienverhältnissen liegt regelmäßig der wichtigste Umstand, der das Einkommen mindert oder den notwendigen Bedarf erhöht. Für die Durchführung des Grundsatzes der gleichmäßigen Besteuerung nach der Leistungsfähigkeit ist daraus abzuleiten, dass die Besteuerung abhängig von der Höhe des freien Einkommens sein muss und auf die „Steuerfähigkeit“ - die Belastung dieses Einkommens - angemessene Rücksicht zu nehmen ist. ${ }^{665}$

\subsection{Die Übereinstimmung des Steuersystems mit den Steuerprinzipien}

Aus der geschichtlichen Entwicklung ist zu entnehmen, dass eine einzige wie auch immer geartete Steuer nicht dazu geeignet ist, den Bedarf eines entwickelten Staates zu decken. Gerade eine Mehrheit und eine Verschiedenartigkeit der Steuern ist eine notwendige Folge der Entwicklung des öffentlichen Bedarfs. Wagner forderte daher eine Kombination von Steuern, „welche die Deckung des Bedarfs unter gleichzeitiger möglichster Berücksichtigung aller oberster Besteuerungsgrundsätze sichert“ . ${ }^{666}$

Dieses Steuersystem müsse zunächst den finanzpolitischen Grundsätzen entsprechen; es müsse ausreichend und beweglich sein. In zweiter Linie müsse die Besteu-

664 Ebd., S. 444.

665 Ebd., S. 447.

666 Ebd., S. 475. 
erung die richtige volks- und einzelwirtschaftliche Steuerquelle treffen und dafür sorgen, dass diese nicht mehr als unvermeidlich beeinträchtigt und die Verteilung des Produktionsertrages nicht in volkswirtschaftlich nachteiliger Weise beeinflusst würde. Erst danach - so Wagner - könne der Ausbau des Steuersystems nach der Richtschnur angegangen werden, welche die Gerechtigkeitsprinzipien vorgeben, zumal diese auch von hohem volkswirtschaftlichen Interesse sind, da sie nicht in sich widersprechender Weise in die Verteilung des Volkseinkommens eingreifen dürften. ${ }^{667}$ Die Postulate, dass die Besteuerung möglichst bestimmt, bequem und mit möglichst kleinen Erhebungskosten verbunden sein soll, stünden im Rang der leitenden Prinzipien an letzter Stelle.

Die Übereinstimmung des Steuersystems mit den Steuerprinzipien ist nach Wagner „etwas Organisches, sich stetig Fortbildendes, das, wie der öffentliche Bedarf und das Wirthschaftsleben selbst, daher auch niemals einen endgültigen Abschluss seiner Entwicklung erreichen kann". 668

\subsection{Die Steuerreformen in den deutschen Staaten und im Deutschen Reich}

\subsubsection{Zur Finanzverfassung im Deutschen Reich}

In der Verfassung des zum 1. Januar 1871 ins Leben getretenen Deutschen Reiches war auch der finanzpolitische Föderalismus geregelt. Zur Wahrnehmung der Aufgaben, die dem Reich oblagen - das waren in erster Linie die Pflege der auswärtigen Beziehungen und die Landesverteidigung -, standen der Reichsregierung die Zolleinnahmen, ein Teil der indirekten Steuern und die Überschüsse aus dem Post- und Telegrafenwesen und der Eisenbahn in Elsass-Lothringen zu. Die Bundesländer und die Gemeinden konnten ihren Finanzbedarf vorwiegend durch das Erheben direkter Steuern decken. Die Reichsregierung hatte bei erhöhtem Finanzbedarf Anspruch auf Matrikularbeiträge der Einzelstaaten, woraus jedoch im Laufe der Jahre Probleme erwuchsen. ${ }^{669}$

Die Matrikularbeiträge, aus der Zeit des Norddeutschen Bundes übernommen, waren auch im Finanzsystem des Deutschen Reiches lediglich als Hilfsleistung und deshalb als zweitrangig gedacht. Die Beiträge waren von Jahr zu Jahr zu beantragen

667 Ebd., S. $484 \mathrm{f}$.

668 Ebd., S. 476.

669 Sahm, Reiner: 5000 Jahre Steuern, S. 242. 
und zu gewähren. Das war eine Einnahmequelle für das Reich, die sich für die Haushaltsgestaltung als unstet und für die Bundesländer als Belastung erwies; denn die Beiträge wurden nach der Einwohnerzahl errechnet und nicht nach der ökonomischen Leistungsfähigkeit der Bundesländer, sodass die Belastungen in den wirtschaftlich schwachen Ländern als ungerecht angesehen wurden. ${ }^{670}$

Otto von Bismarck sprach sich in einer Rede vor dem Reichstag am 22. November 1875 gegen das Fortbestehen dieser Beiträge aus und machte seine steuerpolitischen Ambitionen ${ }^{671}$ wie folgt deutlich:

Ich erkläre mich von Haus aus für indirekte Steuern und halte die direkten für einen harten und plumpen Notbehelf nach Ähnlichkeit der Matrikularbeiträge, mit alleiniger Ausnahme, ich möchte sagen, einer Anstandssteuer, die ich von den direkten immer aufrecht erhalten würde, das ist die Einkommensteuer der reichen Leute, aber wohlverstanden nur der wirklich reichen Leute. Das Ideal[,] nach dem ich strebe, ist möglichst ausschließlich durch indirekte Steuern den Staatsbedarf aufzubringen. ${ }^{672}$

Obwohl sich immer deutlicher abzeichnete, dass die Einnahmen des Reiches nicht mehr ausreichten, um die wachsenden Ausgaben abzudecken, kam noch keine Reichssteuerreform im Bismarck'schen Sinne zustande. Zwischen 1872 und 1877 wurden im Reichstag und im Bundesrat immer wieder direkte und indirekte Steuern vorgeschlagen, die aber aus vielerlei Gründen keine Mehrheit fanden. Dabei handelte es sich um eine Börsen-, Gewerbe- und Stempelsteuer ebenso wie um eine progressive Einkommensteuer. Dagegen hatten sich die Bundesstaaten zur Wehr gesetzt, weil sie befürchteten, dass ihnen der betreffende Anteil entgehen könnte. Gleiches widerfuhr den Steuervorschlägen für Tabak, Rübenzucker, Branntwein und Petroleum. Ebenso kamen aus dem Reichstag Widerstände, dessen parteipolitische Zusammensetzung die Vorhaben nicht begünstigte. Als Ziel bezeichnete Otto von Bismarck später im Reichstag, dass das Reich aus einem „lästigen Kostgänger“ der Bundesstaaten, aus „dem mahnenden Gläubiger“, zu einem „freigebigen Versorger“ werden solle. ${ }^{673}$

670 Ebd., S. 244.

671 Die Reformversuche Ottos von Bismarck waren getragen von dem Bestreben, die Steuerlast von der direkten auf die indirekten Steuern überzuwälzen. Vgl. hierzu Thier, Andreas: Steuergesetzgebung und Verfassung in der konstitutionellen Monarchie. Staatsteuerreformen in Preußen 1871-1893 (Studien zur Europäischen Rechtsgeschichte 119), Frankfurt am Main 1999, S. 129.

672 Zitiert nach Blum, Hans: Das Deutsche Reich zur Zeit Bismarcks. Politische Geschichte von 1871 bis 1890, Leipzig/Wien 1893, S. 172.

673 Ebd., S. 307; Sahm, Reiner: 5000 Jahre Steuern, S. 247. 
Es wirft ein Schlaglicht auf die strukturellen Probleme der Reichsfinanzen, dass sich der Zentralstaat im Rahmen des Finanzföderalismus in wachsendem Umfang verschulden musste, um seine Ausgaben zu finanzieren. Heer und Marine, aber auch Post- und Telegrafenverwaltung benötigten große Summen. Die Reichsschuld vervierfachte sich von 1887 bis 1897, verdoppelte sich noch einmal bis 1913 auf gut 4,9 Mrd. Mark. Insgesamt verdreihundertfachte sich die Reichsschuld zwischen 1877 und $1914 .{ }^{674}$

\subsubsection{Steuergesetzgebung und Staatsfinanzen in Preußen}

\subsubsection{Der Steuerreformprozess ab 1871}

Im Gegensatz zu den Problemen, die sich aus den großen Haushaltslücken beim Deutschen Reich ergaben, konnte der preußische König in der Zeit zwischen 1871 und 1893 aufgrund einer ausgesprochenen günstigen Entwicklung handeln. Die französischen Kriegs-Reparationen sowie der wirtschaftliche Aufschwung („Gründerboom") bescherten dem preußischen Finanzminister volle Kassen. ${ }^{675}$

Die Probleme, mit denen sich der preußische Gesetzgeber konfrontiert sah, waren anderer Art: Zum einen war die preußische Steuerverfassung hoffnungslos veraltet, zum anderen war die Steuerlast extrem ungleich verteilt, was sich aus den sozialen Gegensätzen zwischen Grundbesitz und beweglichem Kapital im Rahmen des steuergesetzgebenden Willensprozesses ergeben hatte. ${ }^{676}$ Durch die fundamentale Veränderung der Wirtschafts- und Sozialstrukturen, die durch die industrielle Revolution ausgelöst worden war, geriet die preußische Steuerverfassung in eine doppelte Schieflage: ${ }^{677}$ Erstens waren die Einkünfte aus beweglichem Kapital gegenüber den Einkünften aus Grund-, Gebäudebesitz und Gewerbe privilegiert. Das Einkommen aus beweglichem Kapital unterlag nur einmal der Personalsteuer, hingegen waren die Grund- und Gebäudebesitzer sowie die Gewerbetreibenden

674 Ullmann, Hans-Peter: Der deutsche Steuerstaat, S. 63.

675 Spoerer, Mark: Steuerlast, S. 68.

676 Thier, Andreas: Steuergesetzgebung und Staatsfinanzen in Preußen 1871-1891, in: Lingelbach, Gerhard (Hrsg.): Staatsfinanzen - Staatsverschuldung - Staatsbankrotte in der europäischen Staaten- und Rechtsgeschichte, Köln/Weimar/Wien 2000, S. 311-313.

677 Ebd., S. 316-323 sowie Ders.: Die Gesetzgebungsverfassung der deutschen konstitutionellen Monarchie als bewegliches System: Preußische Steuergesetzgebung 1879-1893, in: Holtz, Bärbel/ Spenkuch, Hartwin (Hrsg.): Preußens Weg in die politische Moderne. Verfassung - Verwaltung - politische Kultur zwischen Reform und Reformblockade (Berlin-Brandenburgische Akademie der Wissenschaften, Berichte und Abhandlungen 7), Berlin 2001, S. $287 \mathrm{ff}$. 
zusätzlich zu den Personalsteuern einer weiteren Steuer in Form der Grund-, Gebäude- oder Gewerbesteuer unterworfen. Zweitens privilegierte die Verteilung der Steuerlast innerhalb der Personalsteuern die hohen Einkommen, benachteiligte die mittleren Einkommen und überforderte die leistungsschwachen Steuerzahler.

Die preußische Personalsteuer bestand seit $1851^{678}$ aus einer Klassensteuer bis zu einem Jahresseinkommen von 1.000 Talern und einer klassifizierten Einkommensteuer, die bei einem Jahreseinkommen über 1.000 Talern ansetzte. Die Tarifgestaltung war so angelegt, dass bei einem Jahreseinkommen im Umfang von 20.000 Talern eine Steuerquote von 3 Prozent zum Ansatz kam, bei 50.000 Talern wurden diese bei den Steuerpflichtigen lediglich mit 2,4 Prozent beschwert. Ein Steuerpflichtiger mit einem Jahreseinkommen von 1 Million Taler wurde lediglich mit 0,72 Prozent belastet. Während also die hohen Einkommen milde belastet wurden, trugen den größten Teil der Personalsteuerlast die mittelständischen Gewerbetreibenden sowie die mittlere und höhere Beamtenschaft. Ein nicht ganz kleiner Teil der klassensteuerpflichtigen preußischen Bevölkerung war kaum in der Lage, die Klassensteuer zu bezahlen. Von der Klassensteuerpflicht waren lediglich die Empfänger öffentlicher Armenhilfe freigestellt; ein Grundfreibetrag war nicht vorgesehen. Dazu kam der fiskalische Druck durch Zuschläge der preußischen Gemeinden zu den staatlichen Steuern, der die unteren Schichten zusätzlich traf. Diese erhöhten die effektive Belastung der leistungsschwachen Bevölkerung noch einmal drastisch. ${ }^{679}$

Eine weitere Ungleichbehandlung erfolgte dadurch, dass die Bezieher von hohen Einkommen zusätzlich begünstigt wurden, da das Veranlagungsverfahren durch die Schätzung lokaler Kommissionen und nicht durch die Abgabe einer Steuererklärung erfolgte. Während die Einkommen der Beamten relativ einfach zu ermitteln waren, standen den Kommissionen für die Einkünfte aus Grund- und Gebäudebesitz nur veraltete Kataster zur Verfügung. Bei den Einkünften aus beweglichem Kapital fehlte hingegen jede Kontrolle; eine realistische Einschätzung solcher Einnahmen war kaum möglich. ${ }^{680}$

Die veraltete preußische Steuerverfassung sowie die extreme ungleiche Verteilung der Steuerlast bargen erheblichen sozialen und politischen Sprengstoff, weshalb für die politisch Verantwortlichen Handlungsbedarf bestand. Politischer Konsens herrschte über die Notwendigkeit, die Steuerlast bei der einkommensschwachen Bevölkerung zu mindern, was dazu führte, die ausgesprochen unpopuläre Mahlsteuer mit Wirkung zum 1. Januar 1875 aufzuheben, und die bis dahin staatliche

678 Vgl. Kapitel 6.2.3.

679 Thier, Andreas: Steuergesetzgebung und Staatsfinanzen in Preußen 1871-1891, S. 323.

680 Ebd., S. 319. 
Schlachtsteuer den Kommunen zu übertragen. Die seit 1667 bestehende, nur 1810/11 kurz unterbrochene Praxis, Stadt und Land mit unterschiedlichen Staatssteuern zu belasten, war damit beendet. ${ }^{681}$

Diejenigen Einwohner, die in den mahl- und schlachtsteuerpflichtigen Städten wohnten, und deren Jahreseinkommen unter 1.000 Taler betrug, hatten nun die Klassensteuer zu bezahlen. ${ }^{62}$ Die Klassensteuer als auch die klassifizierte Einkommensteuer richtete sich nun nicht mehr nach äußerlichen Merkmalen, sondern nach dem Einkommen. Daneben wurde erstmals ein steuerfreies Existenzminimum in die Klassensteuer eingearbeitet und die Einkommensteuer, die bis dahin bei einem Jahreshöchstbetrag von 7.200 Talern endete, wurde so ausgestaltet, dass sie nach oben offen war. Diese Reform führte zu einer eindeutigen absoluten und relativen Besserstellung der unteren Schichten. Weiterhin wurden die sehr großen Einkommen im gewerblichen und im Dienstleistungssektor belastet, die vor der Reform durch die Veranlagung kaum betroffen waren. ${ }^{683}$

Ein zentrales Element der Steuerreformziele war der Ersatz der preußischen Personalsteuern durch Verbrauchsteuern. Diese sollten auf Reichsebene eingeführt werden, um den Einnahmeausfall der direkten Steuern in Preußen auszugleichen. Otto von Bismarck hatte die reale Gefahr, die dem Gesellschaftssystem aus der Arbeiterbewegung erwachsen konnte, erkannt und war daher bemüht, mit sozialpolitischen Maßnahmen die Arbeiterschaft an das von ihm vertretene Gesellschaftssystem zu binden. Die Steuerreformpolitik wurde damit zu einem Instrument, mit dessen Hilfe gesellschaftliche Probleme beeinflusst werden sollten; sie wurde damit zu einem Teilelement regulierender Staatlichkeit. ${ }^{684}$

Ungelöst blieb jedoch das Problem der ungleichen Steuerlastverteilung zwischen Grundbesitz und Gewerbe und dem beweglichen Kapital. Die Gegensätze zwischen dem ländlichen Grundbesitz und dem städtischen Wirtschaftsbürgertum spiegelten sich größtenteils im sozialen Profil der großen Fraktionen des preußischen Parlaments wider. ${ }^{65}$ Alle großen Fraktionen des Abgeordnetenhauses verband jedoch die

681 Spoerer, Mark: Steuerlast, S.68; vgl. auch Schremmer, Eckart: Finanzreform und Staatshaushalt in Preußen nach 1820. Einige Beurteilungen, in: Ullmann, Hans-Peter/ Zimmermann, Claus (Hrsg.): Restaurationssystem und Reformpolitik. Süddeutschland und Preußen im Vergleich, München, S. 111-138, S. 114.

682 Spoerer, Mark: Steuerlast, S. 68.

683 Ebd., S.71f.

684 Thier, Andreas: Steuergesetzgebung und Staatsfinanzen in Preußen 1871-1891, S. 324.

685 Thier, Andreas: Die Gesetzgebungsverfassung der deutschen konstitutionellen Monarchie als bewegliches System: Preußische Steuergesetzgebung 1879-1893, in: Holtz, Bärbel/ Spenkuch, Hartwin (Hrsg.): Preußens Weg in die politische Moderne. Verfassung Verwaltung - politische Kultur zwischen Reform und Reformblockade (Berlin-Bran- 
Ablehnung von Bismarcks Plänen einer föderalen Steuerreform, was letztendlich zu einem Kompromiss führte. ${ }^{686}$ Am Ende der Kommissionsberatungen stand eine Gesetzesvorlage, die mit einer Einkommen- und Kapitalrentensteuer, der Einführung der Selbsterklärungspflicht, einem progressiven Einkommensteuertarif und der Einführung einer Kapitalertragsteuer wegweisend für die zukünftige preußische Steuerreform sein sollte. ${ }^{687}$

Im Ergebnis setzten sich bei diesem Kompromiss die Repräsentanten des ländlichen Grundbesitzes durch, was sich insbesondere in der Begünstigung der ostelbischen Grundbesitzer bei der Umgestaltung der preußischen Realsteuerverfassung, ${ }^{688}$ aber auch bei der Neuordnung der Einkommen- und Gewerbesteuer zeigte.

\subsubsection{Die Reformgesetzgebung 1890/91 und 1892/93}

Die ersten preußischen Einkommensteuergesetze berücksichtigten in einem nicht unerheblichen Umfang grundlegende finanzpolitische Forderungen, wie sie in der Literatur des 19. Jahrhunderts vorgeschlagen worden waren. Dabei ging es um die schrittweise Verwirklichung des Grundsatzes der Allgemeinheit der Besteuerung, die Einführung eines progressiven Einkommensteuertarifes, die Anerkennung eines angemessenen steuerfreien Existenzminimums, die Berücksichtigung subjektiver Leistungsfähigkeitsmerkmale, wie z. B. die Aufwendungen für den Unterhalt der Familie und die Einführung einer Vermögensteuer, mit deren Hilfe die Einkünfte aus Kapital einer zusätzlichen steuerlichen Belastung unterworfen werden sollten. ${ }^{689}$

Die angewachsene Schuldenlast im preußischen Staat veranlasste Johannes von Miquel - vom preußischen König Wilhelm II. 1890 zum Finanzminister berufen-, eine grundlegende Reform der Einkommensteuer in Angriff zu nehmen. Johannes von Miquel sympathisierte mit den Linken, war Anfang der Fünfzigerjahre ein tätiges Mitglied des Bundes der Kommunisten und begründete 1866 die Nationalliberale Partei mit, die er später an die Konservativen heranführte. ${ }^{600}$ Entscheidend für die

denburgische Akademie der Wissenschaften, Berichte und Abhandlungen 7), Berlin 2001, S. 285-320, S. 296.

686 Thier, Andreas: Steuergesetzgebung und Verfassung, S. 131 und S. 386.

687 Thier, Andreas: Die Gesetzgebungsverfassung, S. 307.

688 Die Realsteuern sollten künftig nicht mehr vom preußischen Staat, sondern durch die Gemeinden erhoben werden, was einem seit Langem vorgetragenen Wunsch aller Fraktionen, den Überlegungen der Finanzwissenschaft und den Interessen der Kommunen entsprach; vgl. Thier, Andreas: Steuergesetzgebung und Staatsfinanzen, S. 331.

689 Davidsohn, Lars: Verfassungsrechtliche Würdigung, S. 164.

690 Hass, Gerhard u.a. (Hrsg.): Biographisches Lexikon zur deutschen Geschichte. Von den Anfängen bis 1945, Berlin 1971, S. 475; Sahm, Reiner: 5000 Jahre Steuern, S. 253. 
seit Langem vorgetragenen Forderungen, Steuerreformen in ein gesetzgebendes Gesamtkonzept umzusetzen, war vor allem der Umstand, dass es von Miquel gelang, einen breiten parlamentarischen Konsens zu finden. ${ }^{691}$

Viele Parlamentarier forderten während der Beratungen zum Preußischen Einkommensteuergesetz 1891 eine Höherbelastung der fundierten Einkommen, doch von Miquel setzte letztlich sein Konzept durch, die Höherbelastung durch die Erbschaft- und Vermögensteuer zu erreichen. ${ }^{692}$ Andreas Thier zählt die Eigentümer ländlichen Grundbesitzes sowie die Unterschichten zu den wichtigsten Gewinnergruppen, hingegen das Wirtschaftsbürgertum als Hauptverlierer der Reformen. ${ }^{693}$

Zwischen 1891 und 1893 vereinigte Johannes von Miquel die Klassen- und klassifizierte Einkommensteuer zu einer modernen Einkommensteuer, die als die erste moderne, systematische deutsche Einkommensteuer gilt. ${ }^{694}$ Diese Einkommensteuer war von der Idee her der entscheidende Schritt in das 20. Jahrhundert. Von Miquel nannte sie bei seiner Rede im Abgeordnetenhaus über die Reform der direkten Steuern vom 20. November 1890 ein „Werk ausgleichender Gerechtigkeit $^{\star{ }^{\prime 695}}$. Von Miquels Ziel war es „eine wirklich gleichmäßige, den heutigen sozialen und wirtschaftlichen Verhältnissen entsprechendere Verteilung der Steuerlast" herbeizuführen. ${ }^{696}$

Eine gleichmäßige und den persönlichen Einkommensverhältnissen angepasste Besteuerung hat von Miquel deshalb als unverzichtbar angesehen, weil eine ungleichmäßige Steuerbelastung „vielfach zu einer Überlastung der geringeren und mittleren Einkommen führt und zu einer Schonung und unberechtigten Bevor-

691 Thier, Andreas: Steuergesetzgebung und Verfassung, S. 434.

692 Richter, Ute G.: Einkommensbesteuerung privater Finanzanlagen in Deutschland, Europa und USA. Ökonomische und rechtliche Gesichtspunkte, Wiesbaden 1995, S. 48.

693 Thier, Andreas: Steuergesetzgebung und Verfassung, S. 970.

694 Oechsle, Klaus: Die steuerlichen Grundrechte, S. 111. Nach einem vorsichtigen Versuch in Hessen im Jahr 1869 stellte Sachsen als erster Bundesstaat sein Steuersystem von den alten objektiven Ertragsteuern auf die neue subjektive Einkommensteuer um. Im Jahr 1874 erschien ein provisorisches, danach 1878 das endgültige Einkommensteuergesetz. Baden erhielt im Jahr 1882 eine Einkommensteuer. Das danach von Preußen eingeführte Steuersystem diente den Einzelstaaten als Vorbild; vgl. Schremmer, Eckart: Steuern und Staatsfinanzen während der Industrialisierung Europas. England, Frankreich, Preußen und das Deutsche Reich 1800 bis 1914, Berlin Heidelberg 1994, S. 204.

695 Miquel, Johannes von: Rede zu der Reform der direkten Steuern im Abgeordnetenhaus am 20.11.1890, S. 329.

696 Miquel, Johannes von: Rede zu den Grundprinzipien der Reform der Einkommensteuer im Abgeordnetenhaus am 16.02.1891, in: Schultze, Walther/Thimme, Friedrich (Hrsg.): Johannes von Miquels Reden, Bd. 3, Halle an der Saale 1913, S. 360-365, S. 365. 
zugung der größeren Einkommen. “697 Da der Staat auf eine gewisse Akzeptanz der Steuerpflichtigen angewiesen sei, seien nachvollziehbare und verständliche Regeln und Prinzipien über eine gerechte Steuerlastverteilung unabdingbar. ${ }^{698}$

Mit diesem Einkommensteuergesetz wurde nun auch der gesamte Adel ab dem 1. April 1893 zur Einkommensteuer veranlagt. ${ }^{69}$ Trotzdem war der Allgemeinheitsgrundsatz der Besteuerung noch nicht vollständig erfüllt, denn nach $₫ 3$ EStG Preußen 1891 waren die Mitglieder des Königlichen Hauses, des Hohenzollerschen Fürstenhauses, das vormalige Hannoversche Königshaus und die vormaligen Kurhessischen und Herzoglich Nassauischen Fürstenhäuser ausdrücklich von der Einkommensteuer befreit. Erst im Jahr 1919 wurden diese letzten absolutistischen Privilegien durch Art. 109 Abs. 3 Satz 1 und Art. 134 der Weimarer Verfassung für unzulässig erklärt und aufgehoben. ${ }^{700}$

Das Einkommensteuergesetz vom 24. Juni $1891^{701}$ berücksichtigte verschiedene objektive und subjektive Leistungsfähigkeitsmerkmale. Die Anzahl der Kinder wurde durch Pauschbeträge ( $\$ 18$ EStG Preußen 1891) ebenso berücksichtigt wie die Geltendmachung von Aufwendungen aufgrund von außergewöhnlichen Belastungen (\$19 EStG Preußen 1891). Insgesamt wurden die unteren Einkommensgruppen entlastet, und der Steuertarif hatte einen gestuft progressiven Charakter.

Nach \$9 EStG (Preußen 1891) waren die zur Erwerbung, Sicherung und Erhaltung des Einkommens verwendeten Ausgaben ebenso zum Abzug zu bringen wie Schuldzinsen und Renten, dauernde Lasten, Abschreibungen und Versicherungen.

Das neue Einkommensteuerrecht unterschied zwischen unbeschränkter und beschränkter Steuerpflicht, zwischen Gewinn- und Überschusseinkünften und wies vier verschiedene Einkunftsarten auf. Eingeführt wurde die Steuererklärungspflicht für alle Einkommen über 3.000 Mark (\$24 EStG Preußen 1891), es erfolgten Änderungen im Veranlagungsverfahren sowie eine Neuregelung des Berufungswesens.

697 Miquel, Johannes von: Die Reform der direkten Steuern, Rede vor dem Abgeordnetenhaus am 20.11.1890, S. $305 \mathrm{f}$.

698 Birk, Dieter: Steuerrecht (Schwerpunkte 3), 6. Aufl., Heidelberg 2003, vor $₫ 1$, Anm. 1 und 5.

$699 \$ 4$ EStG Preußen 1891; vgl. hierzu auch $\$ 1$ des „Gesetzes, betreffend die Aufhebung der Befreiung von ordentlichen Personalsteuern gegen Entschädigung vom 18. Juli 1892“, in: Grotefend, Georg August (Hrsg.): Das gesammte preußisch-deutsche Gesetzgebungs-Material. Die Gesetze und Verordnungen nebst den Erlassen, Reskripten, Anweisungen und Instruktionen der preußischen und deutschen Centralbehörden, Düsseldorf 1892, S. 450.

700 Davidsohn, Lars: Verfassungsrechtliche Würdigung, S. 19.

701 Preußisches Einkommensteuergesetz vom 24. Juni 1891 in: FinanzArchiv 8,2 (1891), S. 331-451. 
Ein eigener unabhängiger Steuergerichtshof wurde in einer Abteilung (Senat) des obersten Verwaltungsgerichtshofes eingerichtet. ${ }^{702}$

Die Steuerpflicht, der bisher nur physische Personen unterlegen waren, wurde auch auf juristische Personen ausgedehnt. Natürliche und juristische Personen wurden im einkommensteuerrechtlichen Sinn völlig gleichgestellt. ${ }^{703}$ Die „Einkommen-Körperschaftsteuer" diente als Ergänzung zur Einkommensteuer, um thesaurierte Gewinne besteuern zu können, die bis dahin nicht von der Einkommensteuer erfasst wurden. Aufgrund der steigenden Steuersätze bei der Einkommensteuer rückte die wirtschaftliche Doppelbelastung ausgeschütteter Gewinne immer mehr ins Bewusstsein und sorgte von Beginn an für grundsätzliche Diskussionen über die Berechtigung und Stellung der Körperschaftsteuer im gesamten Steuersystem. ${ }^{704}$ In den deutschen Bundesstaaten hatten sich zur Verminderung der Doppelbelastung unterschiedliche Körperschaftsteuersysteme herausgebildet, denen jedoch trotz intensiver Begründungsversuche eine gewisse Systemlosigkeit anhaftete. Letztendlich ging es jedem Bundesstaat darum, die sich entwickelnde neue Leistungsfähigkeit von Unternehmen steuerlich zu erfassen. ${ }^{705}$ Eine formale Trennung erfolgte erst durch das Körperschaftsteuergesetz vom 29. März 1920. ${ }^{706}$

Eine Lohnsteuer gab es noch nicht. Bei einem Jahreseinkommen unter 900 Mark entfiel eine Besteuerung. Der Steuersatz bei einem Einkommen zwischen 900 und 1.500 Mark lag bei 0,62 Prozent und wurde bei 3.000 Mark auf 1,73 Prozent angehoben. Höhere Einkommen über 9.500 Mark wurden stärker belastet, der Höchststeuersatz für Einkommen von mehr als 100.000 Mark bis einschließlich 105.000 Mark betrug 4 Prozent und stieg bei höherem Einkommen in Stufen von 5.000 Mark um je 200 Mark ( $\$ 17$ EStG Preußen 1891). ${ }^{707}$

Wer die ihm obliegende Steuererklärung nicht in der in $₫ 30$ EStG (Preußen 1891) gesetzten Frist abgab, musste einen Zuschlag von 25 Prozent der Steuerschuld zahlen. Die Gefahr, bei einer Verkürzung des Steueranspruchs als Steuerhinterzieher

702 Sahm, Reiner: 5000 Jahre Steuern, S. 256.

703 Crezelius, Georg: Steuerrecht II: Die einzelnen Steuerarten, 2. Aufl., München 1994, S. 240 .

704 Brinkmann, Thomas: Die Körperschaftsteuer in der Zeit von 1918 bis 1929, Köln 1987, S. 9.

705 Potthast, Thilo: Die Entwicklung der Körperschaftsteuer von den Vorformen bis zur Unternehmenssteuerreform 2001. Eine Untersuchung körperschaftsteuerlicher Entwicklungstendenzen in Steuergesetzgebung und Steuergestaltung, Frankfurt am Main 2008, S. 26-34.

706 RGBl. 1920, S. 393.

707 Preußisches Einkommensteuergesetz vom 24.06.1891, in: FinanzArchiv 8/2 (1891), S. 331-451. 
entdeckt zu werden, war gering; von den wenigen Strafverfahren wurden mehr als 80 Prozent auf dem Verwaltungsweg erledigt. ${ }^{708}$ Der Finanzwissenschaftler Franz Meisel schätzte, dass bei Einkommen über 3.000 Mark zwischen einem Drittel und der Hälfte der fälligen Steuern hinterzogen wurden. ${ }^{709}$

1893 konnte der preußische Finanzminister eine Vermögensteuer durchsetzen. ${ }^{710}$ Das war ihm früher wegen ihrer „konfiskatorischen“ Wirkung ebenso verwehrt worden wie eine ähnlich konzipierte Erbschaftsteuer. ${ }^{711}$ Von Miquel begründete die Einführung der Vermögensteuer damit, dass aus Gründen der sozialen Gerechtigkeit das fundierte Einkommen höher zu belasten sei als das unfundierte. ${ }^{712}$ Das Einkommensteuergesetz wurde daher im Jahr 1893 durch eine ab $1895 \mathrm{zu}$ erhebende Vermögensteuer ergänzt. Der Besteuerung unterlag das gesamte unbewegliche und bewegliche Vermögen nach Abzug der Schulden.

Diese sogenannte Ergänzungssteuer - sie hatte lediglich eine ergänzende Funktion zur Einkommensteuer - enthielt einen Freibetrag von 6.000 Mark und wurde mit einem proportionalen Steuersatz von 0,5 Prozent versehen, wobei das Vermögen des Haushaltes - darunter auch Schmuck, Kunstgegenstände etc. - nicht erfasst wurde. Ermäßigungen wie z. B. die besondere Belastung des Steuerpflichtigen durch Kinderunterhalt waren vorgesehen. ${ }^{713}$

Die Vermögensteuer war ein hervorragendes Mittel, um die Angaben der Steuerpflichtigen bei der Einkommensteuer zu überprüfen. Da die Finanzverwaltung es weitgehend vermied, die Privatsphäre der Besteuerten zu beleuchten, ${ }^{714}$ konnte auf diesem Weg die Richtigkeit der Angaben der Steuerpflichtigen überprüft werden.

Wie sich die vor dem Ersten Weltkrieg geführte Debatte über Steuerhinterziehung auf das Verhalten der Steuerpflichtigen auswirkte, lässt sich nicht ermitteln. ${ }^{715}$

708 Meisel, Franz: Moral und Technik bei der Veranlagung der preußischen Einkommensteuer, Leipzig 1911, S. 336 f. und S. 347 f.

709 Ebd., S. 285-373.

710 Die Vermögensteuer wurde nach langen Diskussionen akzeptiert, um nicht die Gesamtreform zu gefährden, vgl. Thier, Andreas: Steuergesetzgebung und Verfassung, S. 642.

711 Sahm, Reiner: 5000 Jahre Steuern, S.254.

712 Miquel, Johannes von: Rede zur Vermögensteuer am 17.04.1893, in: Schultze, Walther/ Thimme, Friedrich (Hrsg.): Johannes von Miquels Reden, Bd. 4, Halle an der Saale 1914, S. 65-71, S. 67.

713 Ergänzungssteuergesetz vom 14.07.1893, in: Gesetz-Sammlung für die Königlichen Preußischen Staaten, Jahrgang 1893, S. 134 ff., auf: http://www.verfassungen.de/de/ preussen/ gesetze/erg\%C3\%A4nzungssteuergesetz93.html (letzter Zugriff: 20.02.2017); Sahm, Reiner: 5000 Jahre Steuern, S. $254 \mathrm{f}$.

714 Ullmann, Hans-Peter: Der deutsche Steuerstaat, S. 54.

715 Vgl. hierzu im Einzelnen Ebd., S. 51-54. 
Festgestellt werden kann jedoch, dass es zu einem Umschwung in der öffentlichen Meinung und den Parlamenten kam. Es führte dazu, dass das Wehrbeitrags- und das Vermögenszuwachssteuergesetz 1913 die Kontrollmöglichkeit des Fiskus verschärften und die Steuerhinterziehung nicht nur mit Geldstrafe, sondern jetzt auch mit Freiheitsentzug versehen war. ${ }^{716}$

Da die Einnahmen aus der Einkommensteuer ergiebig waren, konnte in Preußen die Ertragsteuer auf Grundbesitz, Gebäude und Gewerbe den Gemeinden überlassen werden. Das geschah 1895, indem man auch die Gemeindesteuern in die Miquel'sche Reform einbezog, wobei die Gemeinden die Hebesätze wählen konnten. Es gelang freilich nicht, die Grundsteuern durchgreifend zu modernisieren. In den gemeindefreien Gutsbezirken entfiel die Grundsteuer, ein zwischen 9,8 und 11,7 Millionen Mark geschätztes Steuergeschenk an den Großgrundbesitz. ${ }^{71}$

Johannes von Miquel modernisierte auch die Gewerbesteuer. Der Ertrag und das Kapital einer gewerblichen Unternehmung wurden nun Bemessungsgrundlagen. Ein Zwang zur Abgabe der Steuererklärung wurde vermieden; die Gewerbetreibenden brauchten nur anzugeben, in welche der vom Gesetz vorgegebenen vier Klassen der Ertrag ihrer Unternehmung fiel. Während kleine Gewerbebetriebe bis 1.500 Mark steuerfrei blieben, wurde der Steuersatz der obersten Klasse auf 1 Prozent des Ertrags festgesetzt. ${ }^{718}$

Die Miquel'sche Steuerreform wurde lange Zeit als fortschrittlich angesehen. Den Standpunkt, dass durch diese Steuerreform die Steuergerechtigkeit zugenommen habe, sollte jedoch vorsichtiger formuliert werden, als dies in der Forschung in der Vergangenheit geschehen ist. ${ }^{79}$ Andererseits war diese Steuerreform eine herausragende steuer-, gesellschafts- und systempolitische Leistung. ${ }^{720}$

716 Ebd., S. 55.

717 Nipperdey, Thomas: Deutsche Geschichte 1866-1918, Bd. 2: Machtstaat vor der Demokratie, München 1992, S. 176 f.; Sahm, Reiner: 5000 Jahre Steuern, S. 255.

718 Wagner, Adolph: Finanzwissenschaft 3. Teil, S. 370.

719 Vgl. hierzu im Einzelnen Spoerer, Mark: Steuerlast, S. 196.

720 Nipperdey, Thomas: Deutsche Geschichte 1866-1918, S. 176. 


\subsubsection{Die Einkommensteuergesetze der anderen deutschen Staaten}

Die preußische Einkommensteuerreform - aber auch die im Jahr 1893 eingeführte ergänzende Vermögensteuer - hatte für die Einzelstaaten eine Vorbildfunktion. ${ }^{721}$ In den Ländern, die bereits ein Einkommensteuergesetz hatten, wurden die bestehenden Normen nach preußischem Vorbild überarbeitet, in anderen Ländern wurden Einkommensteuergesetze erlassen, die hinsichtlich der Systematik eine nicht zu übersehende Ähnlichkeit mit dem preußischen Gesetz von 1891 aufwiesen. ${ }^{722}$

Die ungleichmäßige Steuerbelastung, die Verschiedenartigkeit der Steuerquellen, aber auch die Mehrbelastung, die der Zentralstaat mit sich brachte, führten dazu, dass die Bundesstaaten die direkte Besteuerung ausbauten, um ihre Einnahmen zu steigern, denn 1881 stammte erst ein knappes Drittel des einzelstaatlichen Steueraufkommens aus solchen Personalsteuern. ${ }^{723}$

Die Einkommensteuersätze machten einen sprunghaften Anstieg aufgrund der Rüstungsausgaben durch den Ersten Weltkrieg. Im Jahr 1913 machten in den Einzelstaaten die Einkommensteuern zusammengenommen 61 Prozent aller Staatssteuern aus. ${ }^{724}$ Bis zum Ersten Weltkrieg schließlich hatten alle Bundesstaaten ein eigenes Einkommensteuergesetz und hatten sich einander angenähert. ${ }^{725}$

\subsubsection{Die Wertzuwachsbesteuerung}

\subsubsection{Die Idee einer Wertzuwachssteuer}

Unter dem Einfluss der bürgerlichen Sozialreform rückten Überlegungen zur Bodengewinnbesteuerung in den Vordergrund, die zwei Jahrhunderte früher entsprungen waren. Schon John Stuart Mill hatte den Standpunkt vertreten, dass der „unverdiente Wertzuwachs" an Grund und Boden einer besonderen Besteuerung zu

721 Lieb, Ralf: Direkte Steuerprogression. Geschichtliche Entwicklung und kritische Würdigung ihrer Begründungen, Wiesbaden 1992, S. $125 \mathrm{ff}$.

722 Oechsle, Klaus: 100 Jahre Einkommensteuergesetze in Deutschland. Ein Beitrag zum preußischen EStG von 1891, in: Steuer und Studium. Zeitschrift für Aus- und Weiterbildung im Steuerrecht 12 (1991), S.203-206, S. 203.

723 Ebd., S. 45.

724 Born, Karl Erich: Wirtschafts- und Sozialgeschichte des Deutschen Kaiserreiches 1867/71-1914, Stuttgart 1985, S. 70 f.

725 Ebd., S.44-46; Popitz, Johannes: Einkommensteuer, in: Elster, Ludwig u.a. (Hrsg.): Handwörterbuch der Staatswissenschaften, Bd. 3, 4. Aufl., Jena 1926, S. 400-491, S. 400. 
unterwerfen sei; denn es entspräche der steuerlichen Gerechtigkeit, dass gleichsam im Schlaf erworbenes Vermögen dem Staate zuzuführen sei. ${ }^{726}$

In Deutschland hatte Johann Heinrich von Thünen (1783-1850) nachzuweisen versucht, dass der Staat einen Teil der Landrente - d.h. den durch den Besitz von Grund und Boden erzielten Gewinn - vereinnahmen dürfe, ohne dass dies die Wirtschaft beeinträchtige. Eine vollständige Vereinnahmung der Landrente lehnte er $\mathrm{ab}$, da dieses die Landwirte von Investitionen in Verbesserungen zurückhalten und damit die kulturellen Fortschritte hemmen würde. ${ }^{727}$

Hermann Heinrich Gossen (1810-1858) empfahl, den in privater Hand befindlichen Grund und Boden durch den Staat aufkaufen zu lassen, um dadurch die Wertsteigerungen der Gesamtheit zuzuführen. Ihm kam es bei diesem Modell jedoch weniger auf die Reform der Finanzen als auf eine Umgestaltung der Wirtschaftsverfassung an. ${ }^{728}$

Großen Einfluss auf die deutsche Bodenreformbewegung hatte der amerikanische Nationalökonom Henry George (1839-1897), der die Bodenrente als Gemeingut ansah und deshalb den Standpunkt vertrat, dass die Wertsteigerung des Grund und Bodens vollständig dem Staat zuzuführen sei. George wollte darüber hinaus das Eigentum an Grund und Boden den Grundbesitzern jedoch nicht entziehen. ${ }^{729} \mathrm{Er}$ war davon überzeugt, dass eine solche Steuer auf die Grundrente so große Erträge abwerfen würde, dass alle übrigen Steuern fortfallen könnten. ${ }^{730}$

Ein maßvolleres und moderateres Steuermodell entwarf Adolf Damaschke (1865-1935) mit einer gestaffelten Bodenwertsteuer, die das „spekulative Zurückhalten deutschen Bodens" von der Bebauung und besten Ausnutzung unmöglich machen sollte. ${ }^{731}$ Zwangsläufig müsse die Grundrente steigen, wenn mit der Zunahme der Bevölkerungsdichte, der industriellen und gewerblichen Entwicklung der

726 Mill, John Stuart: Principles of Political Economy, S. 817 f.

727 Thünen, Johann Heinrich von: Der isolierte Staat in Beziehung auf Landwirtschaft und Nationalökonomie (1842), ND Jena 1910, S. 349.

728 Gossen, Hermann Heinrich: Entwicklung der Gesetze des menschlichen Verkehrs und der daraus fließenden Regeln für menschliches Handeln, 3. Aufl., Berlin 1927, S. 250-273.

729 George, Henry: Fortschritt und Armuth. Eine Untersuchung über die Ursache der industriellen Krisen und der Zunahme der Armuth bei zunehmenden Reichthum, aus dem Englischen von C. D. F. Gütschow, 4. Aufl., Berlin 1890, S. 358 f.

730 Leisner, Walter: Wertzuwachsbesteuerung und Eigentum. Zugleich ein Beitrag zur Lehre vom Leistungseigentum (Schriften zum Steuerrecht 19), Berlin 1978, S. 33 und S. 166.

731 Damaschke, Adolf: Die Bodenreform. Grundsätzliches und Geschichtliches zur Erkenntnis und Überwindung der sozialen Not, 20. Aufl., Jena 1923, S. $474 \mathrm{f}$. 
Städte und den verbesserten Infrastrukturgegebenheiten erhebliche Wertzuwächse zu verzeichnen sind. Was läge also näher, als diese Wertzuwächse einer Besteuerung zu unterwerfen, um die Gegensätze von Arm und Reich auszugleichen und gleichzeitig den Finanzbedarf zu decken?

Unter den deutschen Finanzwissenschaftlern wurden unterschiedliche Ideen zu einer Wertzuwachsteuer vertreten, die von völliger Zustimmung bis zur restlosen Ablehnung reichten. Hans von Nostitz (1863-1958) wollte den unverdienten Wertzuwachs einer steigenden progressiven Besteuerung unterwerfen und begründete dies insbesondere mit den hohen finanziellen Aufwendungen für öffentliche Einrichtungen und die Durchführung von Infrastrukturmaßnahmen. ${ }^{732}$

Johann Viktor Bredt (1879-1940) stand einer Wertzuwachssteuer ablehnend gegenüber, da er in ihr einen Verstoß gegen das Leistungsfähigkeitsprinzip sah. Er hielt eine Beteiligung der öffentlichen Hand an den Wertzuwächsen von Grundeigentümern als Besteuerungsmodell für generell unzulässig und wollte diese mit keiner höheren Besteuerung versehen als das regelmäßige Einkommen. ${ }^{733}$

\subsubsection{Die Wertzuwachsbesteuerung zu Beginn des 20. Jahrhunderts}

Auf kommunaler Ebene hatten bis zum Jahr 1910 insgesamt 652 Gemeinden eine in der Regel von der Wertsteigerung abhängige Grundverkehrsabgabe eingeführt, die jedoch nur den realisierten Wertzuwachs betraf. Die Abschöpfung eines ,unverdienten"Wertzuwachses erfolgte mit dieser Abgabe jedoch nur in geringem Maße, weshalb die Bodenreformer daran glaubten, dass nur mithilfe einer Reichssteuer eine wirksame Besteuerung durchgeführt werden könnte. ${ }^{734}$

Der Gedanke, die Wertzuwachssteuer als Reichssteuer einzuführen, tauchte bei den Beratungen zur Reichsfinanzreform im Jahr 1909 auf. In der Begründung zu diesem Entwurf wurde der Zweck der Steuer wie folgt gekennzeichnet:

Der Abgabe vom Wertzuwachs [...] liegt der Gedanke zugrunde, daß derjenige, der im wesentlichen ohne eigenes Zutun - infolge von Maßnahmen der Gemeinschaft oder anderweit - an seinem Grundbesitz eine Werterhöhung erfahren hat, von dieser

732 Nostiz, Hans von: Wertzuwachssteuer, in: Conrad, Johannes u.a. (Hrsg.): Handwörterbuch der Staatswissenschaften, 3. Aufl., Jena 1911, S. 774-785.

733 Bredt, Johannes Viktor: Die Besteuerung nach der Leistungsfähigkeit. Ein Beitrag zur Systematik und Reform der direkten Steuern in Preußen und dem Reiche, Leipzig 1912, S. 175 und S. $180 \mathrm{ff}$.

734 Bräuer, Karl: Wertzuwachssteuer (Grundstücksgewinnsteuer), in: Elster, Ludwig/Weber, Adolf/Wieser, Friedrich (Hrsg.): Handwörterbuch der Staatswissenschaften, Bd. 8, 4. Aufl., Jena 1928, S. 1017-1042, S. 1017. 
einen Teil an die Gemeinschaft in dem Augenblick abführen soll, in welchem er den Zuwachs in Geld oder Geldeswert umgesetzt erhält. ${ }^{735}$

Das Reichszuwachssteuergesetz ${ }^{736}$ wurde am 14. Februar 1911 verabschiedet und unterwarf den gesamten realisierten Wertzuwachs des Immobilienvermögens einem progressiven Tarif. Der Eingangssteuersatz betrug 10 Prozent und endete bei einem Höchststeuersatz von 30 Prozent, der ab einer Wertsteigerung von 290 Prozent einsetzte.

Dem Allgemeinheitsprinzip der Besteuerung wurde in diesem Gesetz nicht Rechnung getragen, denn der Landesfürst und die Landesfürstin waren ausdrücklich von einer Steuerpflicht befreit worden. ${ }^{737}$

Ein weiteres Gesetz, das den Vermögenszuwachs am Grund-, Betriebs- und Kapitalvermögen erfassen sollte, war das Deutsche Besitzsteuergesetz vom 3. Juli 1913. ${ }^{738}$ Dieses Gesetz, das zusammen mit dem sogenannten Wehrbeitrag ${ }^{739}$ erlassen worden war, löste das Reichszuwachssteuergesetz von 1911 ab. Die Feststellung des Vermögenszuwachses erfolgte erstmals zum 1. April 1917 für den in der Zeit vom 1. Januar 1914 bis zum 31. Dezember 1916 entstandenen Zuwachs, der Vermögenszuwachs sollte danach alle drei Jahre erfasst werden..$^{70}$

Das Gesetz enthielt hohe Freigrenzen. Die Steuer wurde ab einem Zuwachs von über 10.000 Mark erhoben $(\$ 12$ und $\$ 13$ Deutsches Besitzsteuergesetz vom 3 . Juli 1913) und enthielt einen progressiven Tarif, der bei einem Zuwachs von nicht mehr als 50.000 Mark mit 0,75 Prozent des Zuwachses begann und bei Zuwächsen über 1.000.000 Mark 1,5 Prozent betrug. In Abhängigkeit von dem Gesamtwert des steuerbaren Vermögens konnte der Spitzensteuersatz bei einem Wertzuwachs von 10.000.000 Mark eine Höhe von 2,5 Prozent erreichen ( $\$ 25$ Deutsches Besitzsteuergesetz vom 3. Juli 1913).

735 Berckhoff, Ernst: Der Bergbau und das Reichszuwachssteuergesetz, in: Glückauf. Bergund Hüttenmännische Zeitschrift vom 09.11.1912, 48. Jg., Nr. 45, S. 1836-1842.

736 Zuwachssteuergesetz vom 14.02.1911, RGBl. 1911, S. $33 \mathrm{ff}$.

737 Vgl. $\$ 30$ Nr. 1 des Zuwachssteuergesetzes vom 14.02.1911, RGBl. 1911, S. 33.

738 Deutsches Besitzsteuergesetz vom 03.07.1913, RGBl. 1913 Nr. 41, S. 524.

739 Für die Finanzierung der erhöhten Rüstungsanstrengungen wurde der sogenannte Wehrbeitrag - eine einmalige Vermögensabgabe - beschlossen. Die Einführung dieser direkten Steuer - und nicht eine Erhöhung der indirekten Steuern - war dem Umstand zu verdanken, dass die SPD in der Reichstagswahl von 1912 einen beachtlichen Stimmenzuwachs zu verzeichnen hatte; vgl. Gesetz über einen einmaligen außerordentlichen Wehrbeitrag vom 03.07.1913, RGBl. 1913, S. $505 \mathrm{ff}$.

740 Vgl. $\$ 18$ Deutsches Besitzsteuergesetz vom 03.07.1913, RGBl. 1913 Nr. 41, S. 524 ff. 
Mit diesem Gesetz sollte jedoch keine sozialpolitisch umverteilende Abschöpfung des Wertzuwachses vorgenommen werden, sondern es diente der Finanzierung der zunehmenden Militärausgaben. ${ }^{71}$

\title{
7.2.5 Die Erbschaftsteuer
}

\subsubsection{Die Erbschaftsteuer als Instrument einer gezielten Vermögensumverteilung}

Bis in das 19. Jahrhundert hinein hat sich die deutsche Finanztheorie kaum mit Fragen der Besteuerung des Nachlasses auseinandergesetzt. Die Nationalökonomen und Finanzwissenschaftler sahen in einer solchen Steuer einen ,ersten Schritt zum Socialismus und Communismus", einen "Angriff auf Eigenthum und Erbrecht" und die „Grundpfeiler der socialen und wirthschaftlichen Ordnung" gefährdet. ${ }^{742}$

Ab Mitte des 19. Jahrhunderts wurde jedoch der Erbschaftsteuer eine größere Beachtung geschenkt, wobei die Steuersätze noch selten mit progressiver Wirkung nach der Höhe der Erbschaft vorgeschlagen wurden. Viele Nationalökonomen ${ }^{743}$ betrachteten die Erbschaftsteuer

\begin{abstract}
als ein ideales steuerpolitisches Mittel, einen Vermögensausgleich herbeizuführen, denn diese Steuer schien in einem besonderen Maße dazu geeignet, die Besitzunterschiede zu verringern, insbesondere, da durch sie bereits erworbenes Vermögen nochmals einer Besteuerung unterworfen wird. [...] Sozialpolitisch motivierte Ökonomen und Sozialisten hielten sie auch deswegen für eine wichtige und gerechte Steuer, weil das Erbrecht dem Erben regelmäßig unverdientes und müheloses Einkommen verschaffe. ${ }^{74}$
\end{abstract}

Ein hoher Erbschaftsteuersatz wurde daher als ein unverzichtbares Instrument für die Besitz- und Einkommensteuerumverteilung angesehen ${ }^{745}$

Wagner, der sich offen zu dem sozialpolitischen Gesichtspunkt in der Volkswirtschaft und im Recht bekannte, begründete die Erbschaftsteuer damit, dass diese

741 Leisner, Walter: Wertzuwachsbesteuerung, S. $48 \mathrm{f}$.

742 Wagner, Adolph: Finanzwissenschaft 2. Teil, S. 575.

743 Röpke, Wilhelm: Finanzwissenschaft, Berlin/Wien 1929, S. 118-122; Scheel, Friedrich Wilhelm Hans von: Die Erbschaftsteuer, in: Jahrbücher für Nationalökonomie und Statistik 24 (1875), S. 233-261; Wagner, Adolph: Das soziale und ethische Moment in Finanzen und Steuern, in: Die Verhandlungen des vierzehnten Evangelisch-sozialen Kongresses, abgehalten in Darmstadt am 3. und 4. Juni 1903, Göttingen 1903, S.46-79, S. 46.

744 Davidsohn, Lars: Verfassungsrechtliche Würdigung, S.264f.

745 Scheel, Hans von: Die Erbschaftsteuer, S. 233. 
nicht eine eigentliche „Steuer“ sei, sondern ihr Ertrag den Anteil am Volksvermögen darstelle, „den der Staat als Vertreter der Volkskraft seines Erbrechts aus den im Erbesübergang begriffenen Einzelvermögen bezieht. ${ }^{\text {“746 }}$

Er fügte - bezugnehmend auf das Prinzip der Leistungsfähigkeit - noch als spezielle steuerpolitische Begründung hinzu, dass „die Erbschaft für den Erben [...] einen Erwerb oder Vermögensanfall darstellt, welcher demselben ohne Gegenleistung zufällt und die wirtschaftliche Leistungsfähigkeit des neuen Besitzers steigert. "“47 Nach Wagner hat sich die Höhe der Erbschaftsteuer nach dem Verwandtschaftsgrad und nach der absoluten Höhe des Erbanteils zu richten. Bei den nächsten Verwandten sollten niedrige Sätze zum Zuge kommen, die je nach abnehmendem Verwandtschaftsgrad zunehmen und bei den testamentarisch eingesetzten Nicht-Erbberechtigten am Höchsten festgesetzt werden sollten. Ebenso empfahl er auch eine Schenkungsteuer unter Lebenden mit ähnlichen Grundsätzen und einem gleichen Tarif, um Umgehungen des Erbschaftsteuergesetzes zu verhindern. ${ }^{78}$

Für eine progressive Erbschaftsteuer traten auch die Nationalökonomen Friedrich Julius Neumann ${ }^{749}$ und Wilhelm Röpke ${ }^{750}$ ein, welche ebenfalls die Höhe des Nachlasses und den Verwandtschaftsgrad berücksichtigten. Dabei sollte das Vermögen des Erbenden eine besondere Rolle spielen, da in Abhängigkeit von seiner Größe der Steuertarif bestimmt werden sollte.

Der deutsche Publizist Constantin Frantz sah in dem marktwirtschaftlichen Prinzip die Grundlage für die sich steigernde Ungleichheit der Vermögens- und Einkommensverhältnisse und die einzige Lösung dieser Ungleichheit darin, dass von der Staatsgewalt eine Gegenwirkung ausgehe, die sich des Steuerwesens bediene. Das Steuerwesen solle mit direkten Steuern eine ethisch-soziale Funktion ausüben und auf eine bessere Verteilung der Vermögen und Einkommen hinwirken. Unmittelbar dazu dienten nach seiner Ansicht nur die progressive Einkommen- und Erbschaftsteuer, „ohne welche auch alle anderen Steuerformen in socialer Hinsicht fruchtlos bleiben würden. ${ }^{\text {“751 }}$

746 Wagner, Adolph: Finanzwissenschaft 2. Teil, S. 588.

747 Ebd., S. 588.

748 Ebd., S. 592.

749 Neumann, Friedrich Julius: Nach dem Vermögen der Erbenden klassifizierte Erbschaftssteuern, in: Annalen des Deutschen Reichs für Gesetzgebung, Verwaltung und Volkswirtschaft. Rechts- und staatswissenschaftliche Zeitschrift und Materialiensammlung 42 (1909), S. 417-424.

750 Röpke, Wilhelm: Finanzwissenschaft, S. $120 \mathrm{f}$.

751 Frantz, Constantin: Die sociale Steuerreform, S. 51. 
Denn nur die Progressivsteuer kann dahin führen, daß die Hauptlast auf die reichen Klassen fällt [...]. Je höher dann das Gesammtquantum der Steuern ansteigt, um so mehr wird die Vermögens- und Einkommensungleichheit sich vermindern. ${ }^{752}$

Frantz wollte bei der Erbschaftsteuer eine Abstufung nach der Entstehung des Vermögens vornehmen, um insbesondere ihren sozialen Zweck zu erfüllen; „denn von der Entstehungsweise des nachgelassenen Vermögens hängt es eben ab, ob und in wie fern dabei eine Ausbeutung der Gesellschaft anzunehmen sein wird. " $753 \mathrm{Ge}$ rade der ererbte Reichtum habe einen einzigartigen Einfluss auf die Fortdauer der Einkommensunterschiede; deshalb sollte nach Frantz der Tarif der Erbschaftsteuer außer von der Höhe und dem Verwandtschaftsgrad zusätzlich von der Entstehung und der Art des Erwerbs des Nachlasses und der Steuerfähigkeit abhängen. ${ }^{75}$

\subsubsection{Die Erbschaftsteuer im Deutschen Reich}

Am 30. Mai 1873 wurde das erste moderne Erbschaftsteuergesetz in Preußen eingeführt. Mehrere Versuche, ein einheitliches Erbschaftsteuergesetz für das Reich zu erlassen, scheiterten daran, dass in den deutschen Ländern unterschiedliches bürgerliches Recht galt. Erst nachdem das Bürgerliche Gesetzbuch für das gesamte Deutsche Reich eingeführt worden war, war der Weg frei für das Reichserbschaftsteuergesetz vom 3. Juni 1906, das stark vom preußischen Erbschaftsteuergesetz von 1873 beeinflusst war. ${ }^{755}$

Der Grundsatz der Allgemeinheit der Besteuerung wurde auch in diesem Gesetz nicht gewahrt, denn die Erbanfälle an den Landesfürsten und die Landesfürstin wurden von der Steuer befreit. ${ }^{756}$ Eine Besonderheit war auch, dass Ehepartner, Kinder und Enkel von der Steuer grundsätzlich verschont blieben. In $₫ 55$ Abs. 1 ErbStG 1906 wurde erstmals die Steuerpflicht für Schenkungen kodifiziert. Grundlage dazu war die bürgerlich-rechtliche Schenkung, wie sie im zum 1. Januar 1900 in Kraft getretenen Bürgerlichen Gesetzbuch geregelt war.

752 Ebd., S. $84 \mathrm{f}$.

753 Ebd., S. 95.

754 Ebd., S. 86, S.98f. und S. 100.

755 Siegel, Thomas: Geschichte der Erbschaftsteuer (Wissenschaftlicher Aufsatz aus dem Jahr 2012 im Fachbereich Jura - Rechtsphilosophie, Rechtssoziologie, Rechtsgeschichte), München 2012.

756 Vgl. $\$ 13$ des Erbschaftsteuergesetzes vom 03.06.1906, RGBl. 1906, S. 620. 
Neben dem Erbanfallsystem ${ }^{757}$ war das Progressionsprinzip ${ }^{758}$ eingeführt worden. Der Steuertarif bestand aus zwei Teilen, einem progressiven Steuersatz von 4 Prozent bis 10 Prozent in Abhängigkeit vom Verwandtschaftsgrad sowie einem Zuschlag in Abhängigkeit vom Wert des ererbten bzw. geschenkten Vermögens. Nach $\$ 10$ ErbStG 1906 konnte der Höchststeuersatz eine Höhe von insgesamt 25 Prozent erreichen.

\subsection{Zusammenfassung und Fazit}

\section{Zur Verwirklichung des Allgemeinheitsgrundsatzes der Besteuerung}

Obwohl die Frankfurter Paulskirchenverfassung nie in Kraft getreten ist, blieb das Revolutionsjahr 1848 nicht ohne Auswirkungen auf die Steuerverfassungen der einzelnen Länder, wobei die vollständige Verwirklichung des Grundsatzes der Allgemeinheit der Besteuerung erst einige Jahrzehnte später erreicht werden konnte. ${ }^{75}$

Mit dem von Johannes von Miquel entworfenen preußischen Einkommensteuergesetz wurde der gesamte Adel ab dem 1. April 1893 zur Einkommensteuer veranlagt. Trotzdem war der Allgemeinheitsgrundsatz der Besteuerung noch nicht vollständig erfüllt, denn Mitglieder einzelner Königs- und Fürstenhäuser waren nicht nur ausdrücklich von der Einkommensteuer, sondern auch von der Reichszuwachs- und der Reichserbschaftsteuer befreit worden. Erst im Jahr 1919 wurden diese letzten absolutistischen Privilegien durch Art. 109 Abs. 3 Satz 1 und Art. 134 der Weimarer Verfassung für unzulässig erklärt und aufgehoben. Mit der verfassungsrechtlichen Fixierung des Gleichheitspostulats war ein Meilenstein gesetzt worden, der einen Jahrhunderte währenden Streit beendete.

\section{Zum Grundsatz der Gleichmäßigkeit der Besteuerung}

Die Revolution von 1848/49 gab der Theorie der allgemeinen Einkommensteuer neue Kraft und Bedeutung. Mit den großen Umwälzungen des 19. Jahrhunderts, insbesondere der fortschreitenden Industrialisierung, schwand der Glaube, die steuerbaren Objekte anhand äußerlicher Merkmale abschätzen zu können. Dazu kam, dass eine rasch anwachsende Bevölkerungsgruppe zu Lohn- und Gehaltsempfängern wurde und immer mehr Personen ein Zinseinkommen aus Kapitalmarktpapieren

757 Vgl. ebd., $\$ \$ 1,28,29$ Abs. 1.

758 Vgl. ebd., $\$ 10$ Abs. 1 und 2.

759 Davidsohn, Lars: Verfassungsrechtliche Würdigung, S. 18. 
erhielten. Beide Einkunftsarten wurden von den objektiven Ertragsteuern nicht und von den übrigen Steuern nur unzureichend - wenn überhaupt - erfasst. Dies verstieß nicht nur gegen das Gerechtigkeitsprinzip, sondern der Staat sah auch eine neue Einnahmequelle, die er in Anspruch nehmen konnte.

Die Zeit zwischen 1871 und 1893 war durch eine sehr ungleiche Verteilung der Steuerlast gekennzeichnet. Dies schlug sich einerseits in einer Privilegierung der Einkünfte aus beweglichem Kapital gegenüber den Einkünften aus Grund- und Gebäudebesitz sowie Gewerbe nieder. Andererseits benachteiligte die Verteilung der Steuerlast innerhalb der Personalsteuer die mittleren Einkommen und überforderte die leistungsschwachen Bevölkerungskreise.

Diese Ungleichheiten im Steuerrecht bargen erheblichen sozialen und politischen Sprengstoff. Aus dieser Situation heraus wurde die Steuerreformpolitik zu einem Instrument, um gesellschaftliche Probleme zu entschärfen. Mit den preußischen Steuerreformen von 1890/91 und 1892/93 wurde die Steuerrechtsordnung an die veränderten Wirtschafts- und Sozialstrukturen angepasst. Die Reformen berücksichtigten in einem nicht unerheblichen Umfang grundlegende Forderungen, wie sie in der Literatur des 19. Jahrhunderts vorgeschlagen worden waren. Die These zunehmender Steuergerechtigkeit im Deutschland des 19. Jahrhunderts muß jedoch erheblich relativiert werden, denn „Preußen und das Kaiserreich waren politisch rückständige, von alten Eliten dominierte Staaten. Dies zeigte sich auch und gerade im Verhältnis zu ihren steuerzahlenden Bürgern. ${ }^{\text {“760 }}$

\section{Zum Prinzip der Besteuerung nach der Leistungsfähigkeit}

Anfang der Sechzigerjahre des 19. Jahrhunderts begann eine Periode, die sich von allen vorausgehenden Perioden unterschied. Den Anstoß zu einer fundamentalen Veränderung in der gesamten Auffassung des Steuerwesens gaben zwei berühmte Abhandlungen von Albert Schäffle und Gustav Schmoller, die die Steuerpflicht des Menschen, die Person des Staatsbürgers, in den Mittelpunkt der Wirtschafts- und Steuerlehre stellten. Nicht mehr der abstrakte, sondern der wirklich konkrete, von ethischen Motiven bewegte Mensch mit all seinen Bedürfnissen wurde jetzt der wissenschaftlichen Betrachtung zugrunde gelegt. Schäffle wies nach, dass ein Einkommen getrennt von der wirtschaftenden Person nicht denkbar sei, dass es nur durch den Bezug auf den Menschen als solches existiere.

Das von Schäffle und Schmoller aufgestellte „ethische“ Steuerprinzip, wonach jeder nach seiner gesamten ökonomisch-politischen Leistungsfähigkeit das Wohl der Gesamtheit zu fördern verpflichtet sei, wurde in der Folgezeit von zahlreichen

760 Spoerer, Mark: Steuerlast, S. 197. 
Autoren aufgegriffen, die Vorschläge zu einer Weiterentwicklung dieser neuen Lehre machten.

In den Schriften des Vereins für Socialpolitik wurden im Jahr 1873 fünf Gutachten und im Jahr 1877 weitere zehn Gutachten publiziert. Alle Gutachter waren sich über die Zugrundelegung des Leistungsfähigkeitsprinzips als gerechtem Maßstab für Steuern einig. Die Berücksichtigung der Leistungsfähigkeit wurde von den Autoren für notwendig erachtet, weil die auftretenden und sich verschärfenden Einkommens- und Vermögensungleichheiten die soziale und politische Freiheit gefährdeten. Die Autoren akzeptierten fast ausschließlich die progressive Besteuerung und eine stärkere Besteuerung des fundierten Einkommens.

In der Literatur trat eine Erscheinung zutage, die völlig im Gegensatz zur herrschenden Lehre der Besteuerung nach der Leistungsfähigkeit stand: eine Theorie der Ausgabenbesteuerung, die den Verbrauch in den Mittelpunkt stellte. Mehrere Autoren wollten sämtliche Abgaben durch eine einzige proportionale Verbrauchsteuer ersetzen. Als Begründung wurde angeführt, dass die Einkommensteuer zu Ungleichheiten und Bevorzugungen führe und außerdem eine Strafe für Fleiß und Geschicklichkeit darstelle.

Im Königreich Preußen setzte Finanzminister Johannes von Miquel das Prinzip durch, die Steuerpflichtigen nach ihrer persönlich-individuellen Leistungsfähigkeit zu besteuern; die unteren Einkommensgruppen wurden entlastet, und der Steuertarif hatte einen gestuften progressiven Charakter. Berücksichtigt wurden die Anzahl der Kinder ebenso wie außergewöhnliche Belastungen. Die zur Erwerbung, Sicherung und Erhaltung des Einkommens verwendeten Ausgaben waren ebenso zum Abzug zu bringen wie Schuldzinsen, Renten, dauernde Lasten, Abschreibungen und Versicherungen.

\section{Zum Redistributionspostulat oder zum Grundsatz der steuerlichen Umverteilung von Einkommen und Vermögen}

In der zweiten Hälfte des 19. Jahrhunderts eskalierten die sozialen Spannungen in der Bevölkerung. Sowohl die Sozialdemokratische Arbeiterpartei als auch die christlich-soziale Arbeiterpartei traten für eine grundlegende Steuerreform ein, bei der die sozialen Belange eine gewichtige Rolle spielen sollten. Sozialpolitik sollte nicht nur im Geben, sondern auch im Nehmen vollzogen werden und damit auch als Hebel der Gesellschaftsreform dienen.

Adolph Wagner vertrat mit der von ihm postulierten „socialen Funktion der Besteuerung" den Standpunkt, dass eine Abflachung der Wohlstandspyramide durch korrigierende Eingriffe unerlässlich sei, um die sozialen Spannungen abzumildern. Er forderte neben einer stärkeren Progression bei der Besteuerung sowohl 
des Einkommens als auch des Vermögens eine höhere Besteuerung des fundierten Einkommens als des Arbeitseinkommens.

Der überwiegende Teil der zeitgenössischen Staatswissenschaftler stand der Umverteilung von Einkommen und Vermögen jedoch ablehnend gegenüber. Als wesentliche Momente dieser ablehnenden Meinungen wurden die mangelnde Vereinbarkeit mit dem Leistungsfähigkeitsprinzip und die Gefahr einer nicht mehr zu kontrollierenden Willkür genannt.

Obwohl Wagners Lehre von der sozialen Funktion der Besteuerung von der Wissenschaft und Praxis überwiegend abgelehnt worden war, drang sie doch schrittweise in die Steuerpolitik aller fortgeschrittenen Länder ein. Immer mehr wandten sich die Auseinandersetzungen diesem Gerechtigkeitsideal zu. Damit wurde die Steuerpolitik auch als Mittel zur sozialethischen Ausrichtung der Gesellschaftsverfassung betrachtet.

Unter dem Einfluss der bürgerlichen Sozialreform rückten Überlegungen zur Bodengewinnbesteuerung in den Vordergrund, die zwei Jahrhunderte früher entsprungen waren und nunmehr teilweise umgesetzt wurden. Die ersten Ansätze einer steuerlichen Abschöpfung eines unverdienten Wertzuwachses wurden auf kommunaler Ebene verwirklicht. Auch mit dem Reichszuwachssteuergesetz vom 14. Februar 1911 wurde der gesamte realisierte Wertzuwachs des Immobilienvermögens einem progressiven Tarif unterworfen.

Viele Nationalökonomen betrachteten die Erbschaftsteuer als ein ideales steuerpolitisches Mittel, um einen Vermögensausgleich herbeizuführen. Ein hoher Erbschaftsteuersatz wurde als ein unverzichtbares Instrument für die Besitz- und Einkommensteuerumverteilung angesehen. Bezugnehmend auf die Leistungsfähigkeit wurde die Erbschaftsteuer damit begründet, dass die Erbschaft für den Erben einen Vermögensanfall darstellte, welcher ohne Gegenleistung anfalle und damit die wirtschaftliche Leistungsfähigkeit des neuen Besitzers steigere. 
Dieses Buch wird unter der Creative Commons Namensnennung 4.0 International Lizenz (http://creativecommons.org/licenses/by/4.0/deed.de) veröffentlicht, welche die Nutzung, Vervielfältigung, Bearbeitung, Verbreitung und Wiedergabe in jeglichem Medium und Format erlaubt, sofern Sie den/die ursprünglichen Autor(en) und die Quelle ordnungsgemäß nennen, einen Link zur Creative Commons Lizenz beifügen und angeben, ob Änderungen vorgenommen wurden.

Die in diesem Buch enthaltenen Bilder und sonstiges Drittmaterial unterliegen ebenfalls der genannten Creative Commons Lizenz, sofern sich aus der Abbildungslegende nichts anderes ergibt. Sofern das betreffende Material nicht unter der genannten Creative Commons Lizenz steht und die betreffende Handlung nicht nach gesetzlichen Vorschriften erlaubt ist, ist für die oben aufgeführten Weiterverwendungen des Materials die Einwilligung des jeweiligen Rechteinhabers einzuholen.

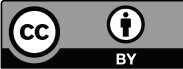

